\title{
Determining the covering factor of compton-thick active galactic nuclei with NuSTAR
}

Brightman, M.; Balokovic, M.; Stern, D.; Areválo, P.; Ballantyne, D. R.; Bauer, F. E.; Boggs, S. E.; Craig, W. W.; Christensen, Finn Erland; Comastri, A.

Published in:

Astrophysical Journal

Link to article, DOI:

$10.1088 / 0004-637 X / 805 / 1 / 41$

Publication date:

2015

Document Version

Publisher's PDF, also known as Version of record

Link back to DTU Orbit

Citation (APA):

Brightman, M., Balokovic, M., Stern, D., Areválo, P., Ballantyne, D. R., Bauer, F. E., Boggs, S. E., Craig, W. W. Christensen, F. E., \& Comastri, A. (2015). Determining the covering factor of compton-thick active galactic nuclei with NuSTAR. Astrophysical Journal, 805(1), [41]. https://doi.org/10.1088/0004-637X/805/1/41

\section{General rights}

Copyright and moral rights for the publications made accessible in the public portal are retained by the authors and/or other copyright owners and it is a condition of accessing publications that users recognise and abide by the legal requirements associated with these rights.

- Users may download and print one copy of any publication from the public portal for the purpose of private study or research.

- You may not further distribute the material or use it for any profit-making activity or commercial gain

- You may freely distribute the URL identifying the publication in the public portal 


\title{
DETERMINING THE COVERING FACTOR OF COMPTON-THICK ACTIVE GALACTIC NUCLEI WITH NUSTAR
}

\author{
M. Brightman ${ }^{1,2}$, M. Baloković ${ }^{1}$, D. Stern ${ }^{3}$, P. Arévalo ${ }^{4}$, D. R. Ballantyne ${ }^{5}$, F. E. Bauer ${ }^{6,7,8}$, S. E. Boggs ${ }^{9}$, \\ W. W. Craig ${ }^{9,10}$, F. E. Christensen ${ }^{11}$, A. Comastri ${ }^{12}$, F. Fuerst $^{1}$, P. Gandhi ${ }^{13,14}$, C. J. Hailey ${ }^{15}$, F. A. Harrison ${ }^{1}$, \\ R. C. Hickox ${ }^{16}$, M. Koss $^{17}$, S. LaMassa ${ }^{18}$, S. Puccetti ${ }^{19,20}{ }^{\text {, E. Rivers }}{ }^{1}$, R. Vasudevan ${ }^{21}$, D. J. Walton ${ }^{1,3}$, and W. W. Zhang ${ }^{22}$ \\ ${ }^{1}$ Cahill Center for Astrophysics, California Institute of Technology, 1216 East California Boulevard, Pasadena, CA 91125, USA \\ ${ }^{2}$ Max-Planck-Institut für extraterrestrische Physik, Giessenbachstrasse 1, D-85748, Garching bei München, Germany \\ ${ }^{3}$ Jet Propulsion Laboratory, California Institute of Technology, 4800 Oak Grove Drive, Mail Stop 169-221, Pasadena, CA 91109, USA \\ ${ }^{4}$ Instituto de Física y Astronoma, Facultad de Ciencias, Universidad de Valparaíso, Gran Bretana N 1111, Playa Ancha, Valparaíso, Chile \\ ${ }^{5}$ Center for Relativistic Astrophysics, School of Physics, Georgia Institute of Technology, Atlanta, GA 30332, USA \\ ${ }^{6}$ Instituto de Astrofísica, Facultad de Física, Pontificia Universidad Catolica de Chile, Casilla 306, Santiago 22, Chile \\ ${ }^{7}$ Millennium Institute of Astrophysics, Chile \\ ${ }_{9}^{8}$ Space Science Institute, 4750 Walnut Street, Suite 205, Boulder, CO 80301, USA \\ ${ }^{9}$ Space Sciences Laboratory, University of California, Berkeley, CA 94720, USA \\ ${ }^{10}$ Lawrence Livermore National Laboratory, Livermore, CA 94550, USA \\ ${ }^{11}$ DTU Space, National Space Institute, Technical University of Denmark, Elektrovej 327, DK-2800 Lyngby, Denmark \\ ${ }_{12}$ INAF Osservatorio Astronomico di Bologna, via Ranzani 1, I-40127 Bologna, Italy \\ ${ }^{13}$ Department of Physics, Durham University, South Road, Durham DH1 3LE, UK \\ ${ }^{14}$ School of Physics \& Astronomy, University of Southampton, Highfield, Southampton SO17 1BJ \\ ${ }^{15}$ Columbia Astrophysics Laboratory, Columbia University, New York, NY 10027, USA \\ ${ }^{16}$ Department of Physics and Astronomy, Dartmouth College, 6127 Wilder Laboratory, Hanover, NH 03755, USA \\ ${ }^{17}$ SNSF Ambizione Fellow, Institute for Astronomy, Department of Physics, ETH Zurich, Wolfgang-Pauli-Strasse 27, CH-8093 Zurich, Switzerland \\ ${ }^{18}$ Yale Center for Astronomy and Astrophysics, Yale University, P.O. Box 208120, New Haven, CT 06520, USA \\ ${ }^{19}$ ASDC-ASI, Via del Politecnico, I-00133 Roma, Italy \\ ${ }^{20}$ INAF-Osservatorio Astronomico di Roma, via Frascati 33, I-00040 Monte Porzio Catone (RM), Italy \\ ${ }_{21}$ Institute of Astronomy, Madingley Road, Cambridge CB3 OHA, UK \\ ${ }^{22}$ NASA Goddard Space Flight Center, Greenbelt, MD 20771, USA \\ Received 2014 November 7; accepted 2015 February 24; published 2015 May 19
}

\begin{abstract}
The covering factor of Compton-thick (CT) obscuring material associated with the torus in active galactic nuclei (AGNs) is at present best understood through the fraction of sources exhibiting CT absorption along the line of sight $\left(N_{\mathrm{H}}>1.5 \times 10^{24} \mathrm{~cm}^{-2}\right)$ in the X-ray band, which reveals the average covering factor. Determining this CT fraction is difficult, however, due to the extreme obscuration. With its spectral coverage at hard X-rays $(>10 \mathrm{keV})$, Nuclear Spectroscopic Telescope Array $(N u S T A R)$ is sensitive to the AGNs covering factor since Compton scattering of X-rays off optically thick material dominates at these energies. We present a spectral analysis of 10 AGNs observed with $N u S T A R$ where the obscuring medium is optically thick to Compton scattering, so-called CT AGNs. We use the torus models of Brightman \& Nandra that predict the X-ray spectrum from reprocessing in a torus and include the torus opening angle as a free parameter and aim to determine the covering factor of the CT gas in these sources individually. Across the sample we find mild to heavy CT columns, with $N_{\mathrm{H}}$ measured from $10^{24}$ to $10^{26} \mathrm{~cm}^{-2}$, and a wide range of covering factors, where individual measurements range from 0.2 to 0.9 . We find that the covering factor, $f_{\mathrm{c}}$, is a strongly decreasing function of the intrinsic $2-10 \mathrm{keV}$ luminosity, $L_{\mathrm{X}}$, where $f_{\mathrm{c}}=(-0.41 \pm 0.13) \log _{10}\left(L_{\mathrm{X}} / \mathrm{erg} \mathrm{s}^{-1}\right)+18.31 \pm 5.33$, across more than two orders of magnitude in $L_{\mathrm{X}}\left(10^{41.5}-\right.$ $\left.10^{44} \mathrm{erg} \mathrm{s}^{-1}\right)$. The covering factors measured here agree well with the obscured fraction as a function of $L_{\mathrm{X}}$ as determined by studies of local AGNs with $L_{\mathrm{X}}>10^{42.5} \mathrm{erg} \mathrm{s}^{-1}$.
\end{abstract}

Key words: galaxies: active - galaxies: individual (NGC 424, NGC 1068, NGC 4945, Circinus) - X-rays: galaxies

\section{INTRODUCTION}

The average covering factor of the obscurer in active galactic nuclei (AGNs), understood to be a torus-like structure, is represented by the ratio of obscured to unobscured AGNs, or the obscured fraction. The obscured fraction has been well studied at many wavelengths, such as the optical (e.g., Lawrence 1991; Simpson 2005), the mid-infrared (e.g., Assef et al. 2013; Gu 2013; Lusso et al. 2013), and the X-rays (e.g., Risaliti et al. 1999), and is largely understood to be dependent on the power of the central source (e.g., Ueda et al. 2003; La Franca et al. 2005; Akylas et al. 2006; Hasinger 2008; Tueller et al. 2008; Beckmann et al. 2009; Akylas \& Georgantopoulos 2009; Brightman \& Nandra 2011b; Burlon et al. 2011; Vasudevan et al. 2013), although some works have shown that this may be an observational effect (e.g., Lawrence \& Elvis 2010; Mayo \& Lawrence 2013).

The fraction of AGNs where the obscuration is so extreme that it is optically thick to Compton scattering $\left(N_{\mathrm{H}}>1.5 \times 10^{24}\right.$ $\mathrm{cm}^{-2}$ ), so-called Compton-thick (CT) AGNs, is less well known (e.g., Brightman \& Nandra 2011b; Burlon et al. 2011), with only a few tens of bonafide CT AGNs known locally (e.g., Goulding et al. 2012; Gandhi et al. 2014). The dependency of the covering factor of CT gas on the power of the AGNs is relatively unknown, even in the local universe, hindered by low number statistics. At the higher redshifts probed by deep extragalactic surveys by Chandra and XMM-Newton, the redshifting of the Compton hump into the bandpasses of these telescopes aids identification of these sources (e.g., Comastri et al. 2011). In addition, the large volumes probed yield of 
order hundreds to thousands of sources overall such that significant numbers of CT AGNs can be uncovered (e.g., Brightman \& Ueda 2012; Brightman et al. 2014; Buchner et al. 2014). Nevertheless, the low count nature of these sources means that constraints on the $N_{\mathrm{H}}$ and $L_{\mathrm{X}}$ are still relatively poor.

The ability to determine covering factors for individual sources is needed to solve many of the issues mentioned above and is needed in order to carry out a detailed study of what physically affects the covering factor. Estimating the covering factor in individual sources can be done by determining the ratio of emission reprocessed in the obscuring medium to the intrinsic emission, which is best done in unobscured sources (i.e., those viewed with a clear line of sight to the nucleus) where the intrinsic emission is directly visible (e.g., Treister et al. 2008; Gandhi et al. 2009; Gu 2013; Lusso et al. 2013). However, the intrinsic emission in such sources can dominate over the reprocessed emission, making them difficult to disentangle, and furthermore disk reflection is difficult to separate from distant reflection. In obscured sources (i.e., those viewed through thick material) the reprocessed emission dominates, though estimates of the intrinsic emission are challenging due to the obscuration itself.

$\mathrm{X}$-ray spectral analysis extending to high energies is ideal for determining the covering factor due to the fact that X-rays above $\sim 3 \mathrm{keV}$ penetrate all but the most extreme obscuration, allowing a good estimate of the intrinsic power. Furthermore, in CT AGNs where the obscuring medium is optically thick to Compton scattering, the scattering of X-rays within the medium can reveal the covering factor of the gas for such sources, especially evident above $10 \mathrm{keV}$. This has been done for a handful of local AGNs, mostly with the use of Suzaku data (e.g., Awaki et al. 2009; Eguchi et al. 2011; Tazaki et al. 2011; Yaqoob 2012; Kawamuro et al. 2013). However, a large statistical analysis on what physically influences the AGN covering factor has yet to be carried out.

The recently launched Nuclear Spectroscopic Telescope Array (NuSTAR; Harrison et al. 2013) is sensitive in the 3-79 keV band. Its significantly improved sensitivity above $10 \mathrm{keV}$ with respect to previous telescopes makes it the ideal instrument to measure the strength and shape of the Compton reflection hump and thus determine the covering factor in a large number of AGNs. As part of its baseline mission, NuSTAR has observed $\sim 100$ AGNs from the hard X-rayselected Swift/BAT survey for $\sim 20 \mathrm{ks}$ each, as well as longer observations of several well-known obscured AGNs such as NGC 1068 and NGC 4945. This sample, which has also been studied at many other wavelengths, is ideal for investigating the covering factor and how it varies. In this paper we present an initial analysis of a small sample of 10 of these sources to investigate how well the covering factor can be determined from X-ray spectra. While this sample is small, it was selected in order to cover a wide range in $L_{\mathrm{X}}$, paying particular attention to low $\left(L_{X} \sim 10^{42} \mathrm{erg} \mathrm{s}^{-1}\right)$ and high $\left(L_{X} \sim 10^{44} \mathrm{erg} \mathrm{s}^{-1}\right)$ luminosity sources to make it as representative as possible. In Section 2, we introduce X-ray spectral torus models and make comparisons between them. In Section 3, we describe the sample and the data analysis. In Section 4, we present our spectral fitting results. In Section 5, we discuss potential biases and systematics. In Section 6, we compare our results to previous results. In Section 7, we explore what physically influences the AGN covering factor. Finally, in Section 8, we present our conclusions. We assume a flat cosmological model with $H_{0}=70 \mathrm{~km} \mathrm{~s}^{-1} \mathrm{Mpc}^{-1}$ and $\Omega_{\Lambda}=0.73$. For measurement uncertainties on our spectral fit parameters we present the $90 \%$ confidence limits given two interesting parameters $(\Delta \chi=4.61)$.

\section{X-RAY SPECTRAL TORUS MODELS}

We first briefly summarize the development of X-ray spectral torus models, detailing their similarities and differences. We then illustrate the differences in the X-ray spectra resulting from these models by fitting simulated spectra created from one model to other sets of public models and comparing the results.

\subsection{Model Details}

In recent years a suite of new X-ray spectral models that describe the reprocessing of X-rays in a torus-shaped medium have been published (Ikeda et al. 2009; Murphy \& Yaqoob 2009; Brightman \& Nandra 2011a; Liu \& Li 2014). These models employ Monte Carlo techniques to calculate spectra, simulating photoelectric absorption, fluorescence, and Compton scattering, and build on previous work by Matt et al. (1991), Leahy \& Creighton (1993), Ghisellini et al. (1994), Nandra \& George (1994), and Yaqoob (1997). The aforementioned models differ mostly in the geometry of the torus considered and the treatment of the different components, be it direct, scattered, or line emission. The underlying physics of the models is the same, however, with photoelectric cross sections from Verner et al. (1996), abundances from Anders \& Grevesse (1989), and fluorescent yields from Bambynek et al. (1972). However, the physical geometry and treatment of Compton scattering differ somewhat. The latest edition from Liu \& Li (2014) is the first to explicitly simulate a clumpy torus.

The model of Ikeda et al. (2009) considers a spherical torus, which consists of a spherical distribution of matter with a biconical void. The column density, $N_{\mathrm{H}}$, through the torus is a function of the inclination angle, where the $N_{\mathrm{H}}$ is maximum when the torus is seen edge-on $\left(90^{\circ}\right.$ inclination angle). This decreases to zero as the inclination angle decreases and reaches the opening angle, at which point the source can be seen unobscured. The opening angle is a free parameter with a range of $0^{\circ}-70^{\circ}$. The model separates into three components: the direct zeroth-order transmitted component, the absorbed reflected component, and the unabsorbed reflected component.

Murphy \& Yaqoob (2009) consider a torus with a circular cross section and a fixed opening angle of $60^{\circ}$ (covering factor of 0.5 ). This model, known as MYTORUs, also has separate components for the direct, scattered, and line emission, motivated so that time variability between these different emissions can be studied. The line components are separated so as to account for instrumental systematic effects on the line energies. Both MYTORUS and Ikeda et al. (2009) consider $N_{\mathrm{H}}$ up to $10^{25} \mathrm{~cm}^{-2}$.

A new implementation of MYTORUs was introduced by Yaqoob (2012) in which the various model components can be used independently in so-called decoupled mode, giving it added flexibility. In this way, it can be used to model a clumpy distribution of matter with an arbitrary covering factor.

The model of Brightman \& Nandra (2011a) also assumes a spherical torus with a biconical void, as in Ikeda et al. (2009). However, the line-of-sight $N_{\mathrm{H}}$ through the torus is constant and 


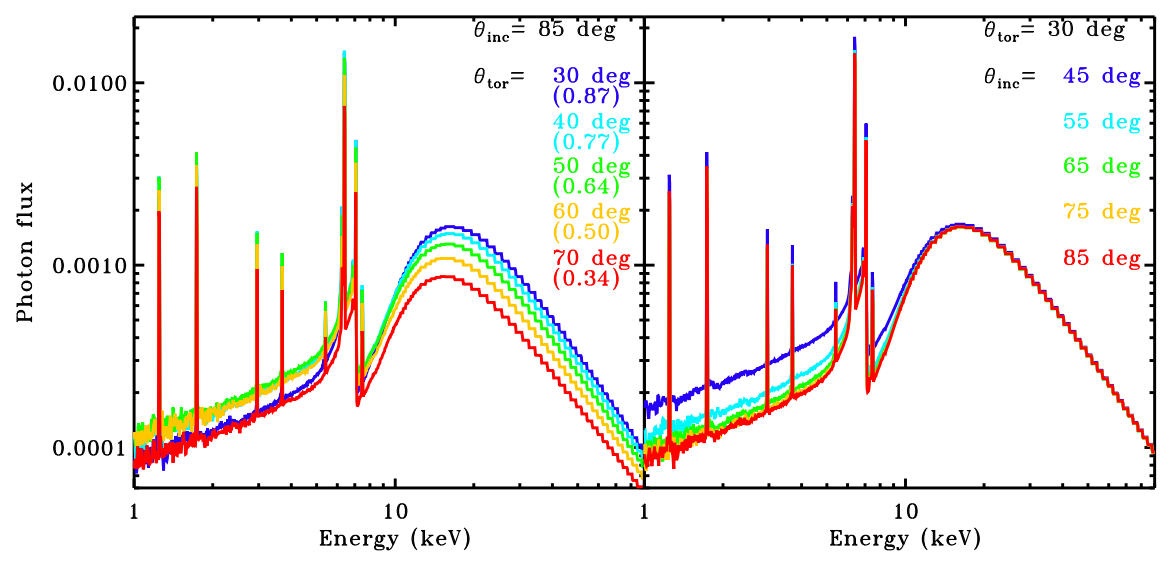

Figure 1. X-ray TORUs spectra for $N_{\mathrm{H}}=2 \times 10^{24} \mathrm{~cm}^{-2}, \Gamma=2$, and different torus opening angles, $\theta_{\text {tor }}($ left), where the equivalent covering factor is in parentheses, and different inclination angles, $\theta_{\text {inc }}$ (right). The variation in spectral shape with $\theta_{\text {tor }}$ above $10 \mathrm{keV}$ is clear, illustrating the capability of NuSTAR to determine the torus opening angle for CT AGNs. For a given torus opening angle, changes in the inclination angle primarily affect the $<10 \mathrm{keV} \mathrm{X}$-ray spectrum.

not dependent on the inclination angle. This model also allows for a variable opening angle, ranging from $26^{\circ}$ to $84^{\circ}$, and has the added advantage of extending up to $N_{\mathrm{H}}=10^{26} \mathrm{~cm}^{-2}$, thus allowing investigations of the most extreme obscuration. We refer to this model simply as the ToRus model. In addition to this TORUs model, Brightman \& Nandra (2011a) present a model for the special case where the source is fully covered (a spherical geometry) and includes variable elemental and iron abundances with respect to hydrogen. We refer to this model as the SPHERE model.

Recently Liu \& Li (2014) have investigated the effect of a clumpy medium, distributed in a toroidal geometry, on the emergent X-ray spectra. The parameters they consider are the volume filling factor of the clumps, the number of clumps along the line of sight, and the line-of-sight $N_{\mathrm{H}}$. They also only consider $N_{\mathrm{H}}$ up to $10^{25} \mathrm{~cm}^{-2}$.

In our investigation of the covering factor in CT AGNs, we use the Brightman \& Nandra (2011a) TORus model because it includes a variable torus opening angle and extends to a higher $N_{\mathrm{H}}$ range. Henceforth we will refer to the half-opening angle of the torus, measured from the polar axis to the edge of the torus, as $\theta_{\text {tor }}$. The covering factor of the torus is simply calculated from $\theta_{\text {tor }}$ following $f_{\mathrm{c}}=\cos \left(\theta_{\text {tor }}\right)$. Figure 1 shows spectra from this model, where the left panel shows a range of $\theta_{\text {tor }}$ values (equivalent to a range of $f_{\mathrm{c}}$ values), and the right panel shows a range in inclination angles $\left(\theta_{\text {inc }}\right)$. The effect of the variation of the opening angle can clearly be seen in the left panel over the entire 3-79 keV spectral range of NUSTAR, in particular in the strength of the Compton hump at $\sim 20 \mathrm{keV}$. This illustrates how NUSTAR is well suited for studying the covering factor in CT AGNs.

\subsection{Model Comparisons}

As these X-ray torus models are still fairly novel, interpreting their results when applied to real data is not yet fully tested or understood. In order to interpret our results using the TORUS model and to make inferences from them, we aim to better discern how this model relates to other commonly used models. We therefore compare the TORUs model to the PEXRAV model of Magdziarz \& Zdziarski (1995), which describes the Compton reflection of X-rays off a semi-infinite slab of cold material. The PEXRAV model has been extensively used in the literature for describing the effect of Compton scattering/ reflection in AGN spectra and for fitting the spectra of reflection-dominated, CT AGNs. Furthermore, we compare the TORUS model to MYTORUs, described above. These two models have differing geometries, and thus a comparison highlights the effects of geometry on the X-ray spectra. MYTORUs has been used frequently in the analysis of NUSTAR spectra of heavily obscured AGNs (e.g., Arévalo et al. 2014; Baloković et al. 2014; Bauer et al. 2014; Gandhi et al. 2014; Puccetti et al. 2014), and thus a comparison is valuable. We do not make a comparison to the Ikeda et al. (2009) model or the clumpy torus models of Liu \& $\mathrm{Li}$ (2014) as their models are not public.

To make the comparisons, we simulate NUSTAR spectra from the TORus model for $N_{\mathrm{H}}=10^{24}, 10^{25}$, and $10^{26} \mathrm{~cm}^{-2}$ and for $\theta_{\text {tor }}=40^{\circ}, 60^{\circ}$, and $80^{\circ}$ for $\Gamma=1.9$ and a $3-79 \mathrm{keV}$ flux of $\sim 10^{-11} \mathrm{erg} \mathrm{cm}^{-2} \mathrm{~s}^{-1}$. We simulate NUSTAR spectra with $100 \mathrm{ks}$ exposures without counting statistics in order to only consider the effect of the model parameters on the spectrum, and we use response and background files from a randomly selected observation, that of NGC 1320 (see Table 1, obsID 60061036004). As a consistency check, we fit the torus model to the simulated data, where we find that the input parameters are recovered.

We subsequently fit each simulated spectrum between 3 and $79 \mathrm{keV}$ with the pure reflection component of PEXRAV with the high-energy cutoff in the continuum set to the maximum $\left(1 \times 10^{6} \mathrm{eV}\right)$, the abundances at solar, and the cosine of the inclination angle at 0.45 . We add a narrow Gaussian component at $6.4 \mathrm{keV}$ to model the $\mathrm{Fe} \mathrm{K} \alpha$ line, since, unlike TORUS, PEXRAV does not self-consistently include Fe fluorescence. For $N_{\mathrm{H}}=10^{24} \mathrm{~cm}^{-2}$, an additional absorbed power-law component is required, which we model with ZWABS $\times$ POWERLAW, the photon index of which is fixed to that of PEXRAV. Figure 2 shows how the best-fit model compares to the simulated spectra. For torus $N_{\mathrm{H}}=10^{24} \mathrm{~cm}^{-2}$, where the optical depth to Compton scattering is just below unity, the fitted models agree with the ToRus model, with no obvious deviations from unity in the data-to-model ratio. This is also the case for all $N_{\mathrm{H}}$ values with a $60^{\circ}$ opening angle. However, at small $\left(40^{\circ}\right)$ and large $\left(80^{\circ}\right)$ opening angles, when fitting with PEXRAV there are obvious deviations in the data-to-model ratio above $10 \mathrm{keV}$. This implies that PEXRAV is not able to reproduce the varying high-energy spectral shapes produced by a torus geometry. 
Table 1

Summary of the Observational Data Used in This Analysis

\begin{tabular}{|c|c|c|c|c|c|c|c|}
\hline Source name & $\begin{array}{l}\text { R.A. } \\
\text { (deg) }\end{array}$ & $\begin{array}{l}\text { Decl. } \\
\text { (deg) }\end{array}$ & Telescope & obsID & Date & $\begin{array}{l}\text { Exposure } \\
\quad(\mathrm{ks})\end{array}$ & $\begin{array}{l}\text { Net Count Rate } \\
\left(\text { counts s }{ }^{-1}\right)\end{array}$ \\
\hline (1) & $(2)$ & (3) & (4) & (5) & (6) & (7) & (8) \\
\hline NGC 424 & 17.86511 & -38.08347 & NuSTAR & 60061007002 & 2013 Jan 26 & 15.5 & $0.052 / 0.053$ \\
\hline$\ldots$ & $\ldots$ & $\ldots$ & XMM-Newton & 2942301 & 2001 Dec 10 & 4.5 & 0.236 \\
\hline NGC 1068 & 40.66963 & -0.01328 & NuSTAR & 60002030002 & 2012 Dec 18 & 57.8 & $0.112 / 0.107$ \\
\hline$\cdots$ & $\ldots$ & $\ldots$ & $\ldots$ & 60002030004 & 2012 Dec 20 & 48.6 & $0.111 / 0.111$ \\
\hline$\cdots$ & $\ldots$ & $\ldots$ & $\ldots$ & 60002030006 & 2012 Dec 21 & 19.5 & $0.115 / 0.113$ \\
\hline 2MFGC 2280 & 42.67746 & 54.70489 & NuSTAR & 60061030002 & 2013 Feb 16 & 15.9 & $0.019 / 0.018$ \\
\hline NGC 1320 & 51.20292 & -3.04228 & NuSTAR & 60061036002 & 2012 Oct 25 & 14.5 & $0.028 / 0.023$ \\
\hline$\cdots$ & $\ldots$ & $\ldots$ & $\ldots$ & 60061036004 & 2013 Feb 10 & 28.0 & $0.025 / 0.022$ \\
\hline$\cdots$ & $\cdots$ & $\ldots$ & XMM-Newton & 405240201 & 2006 Aug 06 & 12.5 & 0.081 \\
\hline NGC 1386 & 54.19242 & -35.99941 & NuSTAR & 60001063002 & 2013 Jul 19 & 21.2 & $0.010 / 0.011$ \\
\hline$\cdots$ & $\ldots$ & $\ldots$ & XMM-Newton & 140950201 & 2002 Dec 29 & 13.9 & 0.153 \\
\hline NGC 3079 & 150.49085 & 55.67979 & NuSTAR & 60061097002 & 2013 Nov 12 & 21.5 & $0.062 / 0.063$ \\
\hline$\cdots$ & $\ldots$ & $\ldots$ & XMM-Newton & 110930201 & 2001 Apr 13 & 13.6 & 0.092 \\
\hline IC 2560 & 154.07799 & -33.56379 & NuSTAR & 50001039002 & 2013 Jan 28 & 23.4 & $0.012 / 0.012$ \\
\hline$\cdots$ & $\ldots$ & $\ldots$ & $\ldots$ & 50001039004 & 2014 Jul 16 & 49.6 & $0.012 / 0.012$ \\
\hline$\cdots$ & $\cdots$ & $\ldots$ & XMM-Newton & 0203890101 & 2003 Dec 26 & 80.5 & $0.006 / 0.007$ \\
\hline Mrk 34 & 158.53580 & 60.03111 & NuSTAR & 60001134002 & 2013 Sep 19 & 23.9 & $0.007 / 0.007$ \\
\hline$\cdots$ & $\ldots$ & $\ldots$ & $X M M-N e w t o n$ & 0306050701 & 2005 Apr 04 & $8.8 / 22.9$ & $0.070 / 0.013$ \\
\hline NGC 4945 & 196.36449 & -49.46821 & NuSTAR & 60002051002 & 2013 Feb 10 & 45.2 & $0.263 / 0.249$ \\
\hline Circinus & 213.29146 & -65.33922 & NUSTAR & 60002039002 & 2013 Jan 25 & 53.8 & $1.046 / 0.984$ \\
\hline
\end{tabular}

Note. Column (1) gives the source name, Columns (2) and (3) list the J2000 position given in NED in degrees, column (4) gives the telescope name, column (5) lists the observation ID, column (6) gives the start date of the observation, column (7) gives the exposure time in ks, and column (8) gives the net count rate for each instrument, be it FPMA/B for NuSTAR (3-79 keV) or pn/MOS for XMM-Newton (0.5-10 keV). Only pn data are used for NGC 424, NGC 1320, NGC 1386, and NGC 3079, and only MOS data are used for IC 2560.

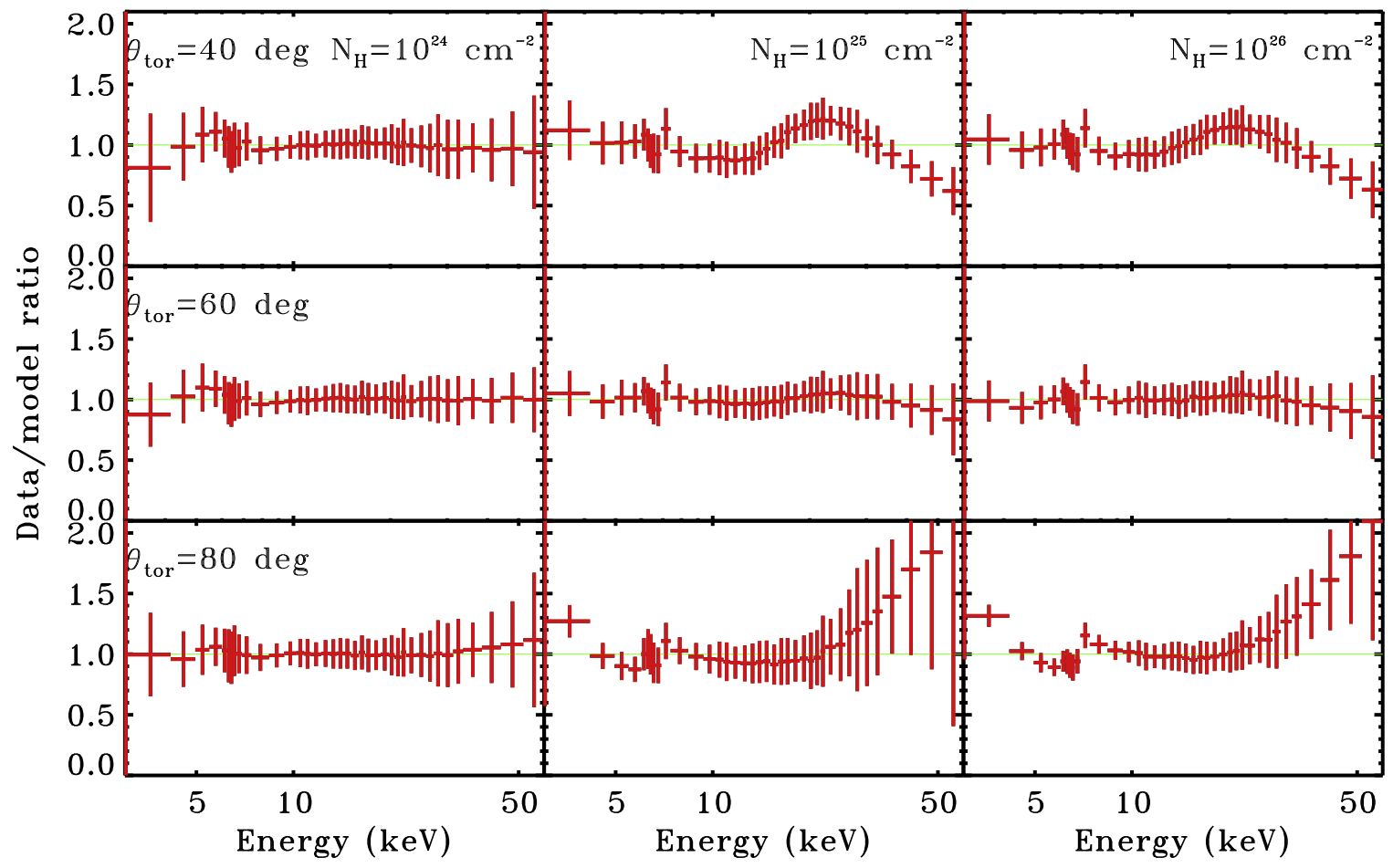

Figure 2. Data-to-model ratios of simulated NuSTAR spectra using the TORUS model (red data points) fitted by PEXRAV plus an absorbed power law for $N_{\mathrm{H}}=10^{24}$ $\mathrm{cm}^{-2}$, for three torus $N_{\mathrm{H}}$ values $\left(N_{\mathrm{H}}=10^{24}, 10^{25}\right.$, and $\left.10^{26} \mathrm{~cm}^{-2}\right)$ and three values of $\theta_{\text {tor }}\left(40^{\circ}, 60^{\circ}\right.$, and $\left.80^{\circ}\right)$. For torus $N_{\mathrm{H}}=10^{24} \mathrm{~cm}^{-2}$, where the optical depth to Compton scattering is just below unity, the fitted models agree with the ToRus model. This is also the case for all $N_{\mathrm{H}}$ values with a $60^{\circ}$ opening angle. However, at small $\left(40^{\circ}\right)$ and large $\left(80^{\circ}\right)$ opening angles, PEXRAV cannot reproduce the shape of the torus model.

Following this, we fit MYTORUS to these simulated spectra in both coupled and decoupled mode. In coupled mode the parameters of the scattered, direct, and line components are fixed to one another, and thus the covering factor is 0.5 . For the decoupled mode we combine the direct absorbed component with two scattered and line components, one where the 


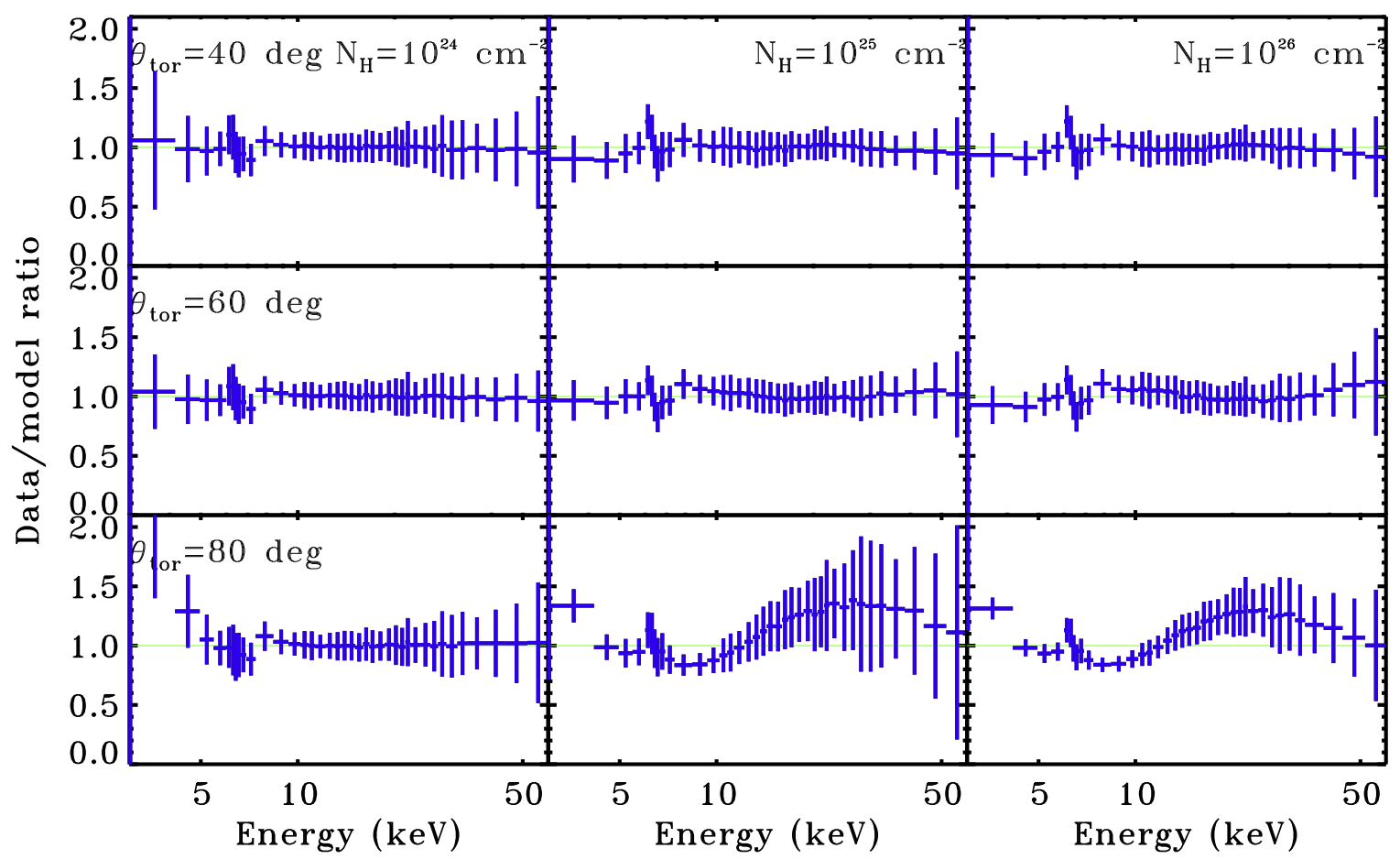

Figure 3. Same as Figure 2, but simulated spectra fitted by MYTORus in decoupled mode.

inclination is fixed to $0^{\circ}$, the other where it is fixed to $90^{\circ}$, with all parameters linked with the exemption of the normalizations. This implementation represents backward scattering and forward scattering, respectively, which, as described by Yaqoob (2012), mimics an absorber with a clumpy distribution of matter with a variable covering factor. The data-to-model ratios for the fit with MYTORUs in decoupled mode are shown in Figure 3. These show that the flexibility of MYTORus in this mode can reproduce the spectral shape of the TORUs model for a wide range of parameters, showing deviations only for the extreme case of $\theta_{\mathrm{tor}}=80^{\circ}$ and $N_{\mathrm{H}}=10^{25} \mathrm{~cm}^{-2}$ and above.

Since the power-law index, $\Gamma$, is a good indicator of the overall shape of the spectrum, we compare that parameter for the fitted models to the value of $\Gamma$ adopted by the input model. We do not aim to test if the fitted models can recover the intrinsic $\Gamma$, since the models have different geometries; rather, we explore the differences in $\Gamma$ in order to gain insight into the various spectral shapes produced by each model. This will also allow better interpretation of the parameters obtained when fitting these models to real data. Figure 4 shows how the bestfit $\Gamma$ from PEXRAV and MYTORUS compare to the input value of 1.9 from the TORUS model given the input $N_{\mathrm{H}}$ and $\theta_{\text {tor }}$ parameters. For all models, the measured $\Gamma$ is consistent with the input $\Gamma$ at $N_{\mathrm{H}}=10^{24} \mathrm{~cm}^{-2}$. For PEXRAv, this diverges to lower $\Gamma$ values at higher $N_{\mathrm{H}}$ for $40^{\circ}$ and $60^{\circ}$ opening angles, indicating that the TORUs model is producing a stronger Compton hump than PEXRAV for these parameters. MYTORUS in coupled mode is roughly consistent at $40^{\circ}$, whereas $60^{\circ}$ shows no difference. This is expected since the opening angle in this model is indeed $60^{\circ}$. For $80^{\circ}$, both PEXRAV and MYTORUS measure rather larger $\Gamma$ values, indicating that the Compton hump is weaker in the TORUs model for large $\theta_{\text {tor }}$.

For MYTORUS in decoupled mode, as in coupled mode, the measured $\Gamma$ from the simulated spectra agree with the input value within the measurement uncertainties for the case of $40^{\circ}$ and $60^{\circ}$. The measurement uncertainties are higher in decoupled mode due to the larger number of degrees of freedom with this implementation. For the case of $80^{\circ}$ there is a large discrepancy between the input and recovered $\Gamma$ values.

As described above, the decoupled implementation of MYTORUS allows for an arbitrary covering factor. Following Yaqoob (2012), Puccetti et al. (2014) estimated the covering factor for NGC 4945 from $f_{\mathrm{c}} \sim 0.5 \times A_{\mathrm{S} 90} / A_{\mathrm{Z} 90}$, the ratio of the normalizations of the direct and scattered components at $90^{\circ}$ inclination angles. We can directly compare this estimation with our input covering factors. Our input $\theta_{\text {tor values }}$ correspond to $f_{\mathrm{c}}=0.77,0.50$, and 0.17 . From the above formulation, we find the covering factors estimated by the decoupled implementation of MYTORUs to be $0.41,0.09$, and 0 , respectively, which are much lower than the input values. It does, however, recover the input trend of decreasing covering factors. The decoupled implementation of MYTORUs only mimics a free covering factor, and thus an agreement was not necessarily expected.

Along with the PEXRAV deviations in spectral shape shown in Figure 2, the above analysis has shown that PEXRAV will also systematically obtain different spectral parameters compared to the TORus model. We have also shown that the opening angle is an important parameter in determining the spectral shape, and thus models with fixed opening angles, such as MYTORUs, will also systematically obtain different parameters. We therefore conclude that slab reflection models should not be used for fitting the high-energy X-ray spectra of CT AGNs, as concluded by Murphy \& Yaqoob (2009), and that ideally spectral models with the covering factor of the CT gas as a free parameter should be used.

\section{SAMPLE PROPERTIES AND DATA ANALYSIS}

Our sample consists of 10 local $(z<0.1)$ CT AGNs observed by NuSTAR: NGC 424, NGC 1068, 2MFGC 2280, 


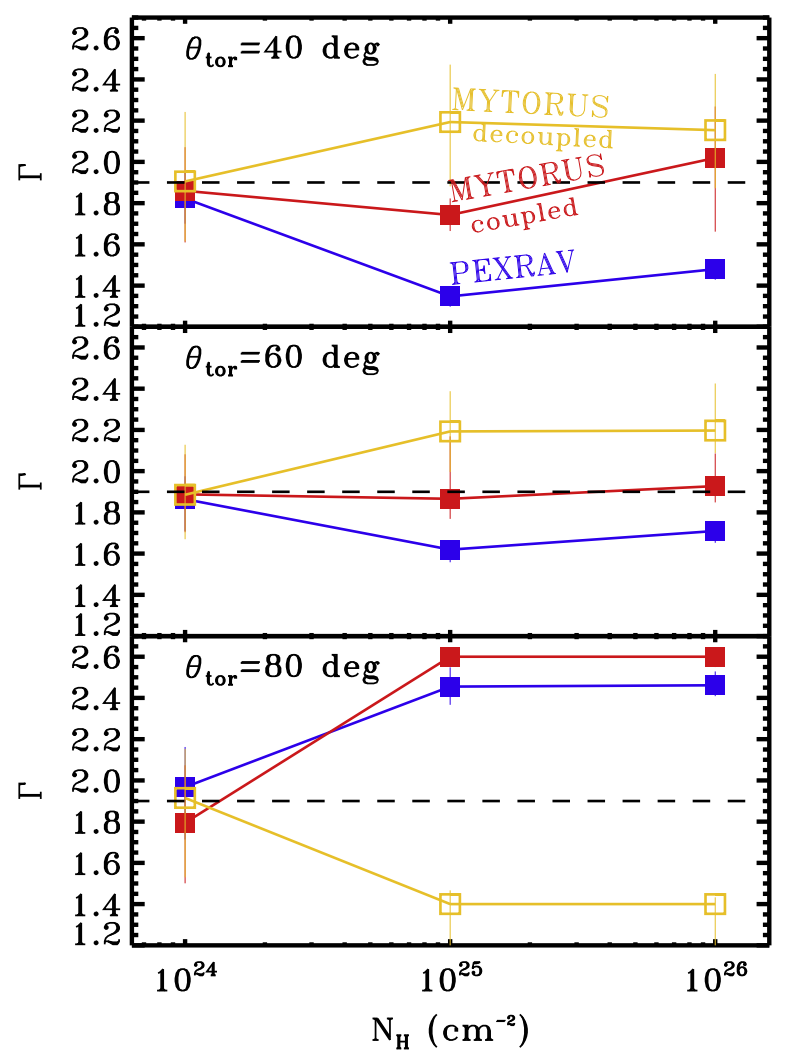

Figure 4. Comparison of the power-law index, $\Gamma$, determined from fitting PEXRAV and MYTORUS to simulated NUSTAR spectra using the TORUS model ( $\Gamma=1.9$, dashed line) for $N_{\mathrm{H}}=10^{24}, 10^{25}$, and $10^{26} \mathrm{~cm}^{-2}$ and three opening angles $\left(40^{\circ}, 60^{\circ}\right.$, and $\left.80^{\circ}\right)$.

NGC 1320, NGC 1386, NGC 3079, IC 2560, Mrk 34, NGC 4945, and the Circinus galaxy. With the exception of 2MFGC 2280, these sources were selected to be known CT AGNs and to cover a wide range in X-ray luminosity. Particular attention was paid to selecting sources in the low $\left(L_{\mathrm{X}} \sim 10^{42} \mathrm{erg} \mathrm{s}^{-1}\right)$ and high $\left(\sim 10^{44} \mathrm{erg} \mathrm{s}^{-1}\right)$ luminosity regimes, thus enveloping the bulk of the local CT AGNs population, which is at moderate luminosities, so that our sample could be as representative as possible, despite its small size. CT AGNs are selected since the obscuration is required to be optically thick to Compton scattering in order for the models to be effective at distinguishing the covering factor. The models are degenerate with covering factor for Compton-thin media. Previously published NuSTAR observations were included in order to facilitate comparison among models.

Observations of NGC 424, NGC 1320, and IC 2560 are presented in Baloković et al. (2014) with modeling using PEXRAV and MYTORUS. Results on NGC 1068 are presented in Bauer et al. (2014), also with modeling using PEXRAV and MYTORUS. Mrk 34 is presented in Gandhi et al. (2014) with modeling using MYTORUs and TORUS. NGC 4945 is presented in Puccetti et al. (2014), and Circinus is presented in Arévalo et al. (2014); both are modeled using MYTORUS. The NuSTAR observations of NGC 1386, NGC 3079, and 2MFGC 2280 are presented here for the first time. No detailed X-ray spectral modeling has previously been published on 2MFGC 2280 and it was selected for study here based on its flat spectral shape in the NUSTAR band, which resembles that of the other obscured AGNs considered in this work.
Table 1 summarizes the basic observational data for this sample. The NUSTAR observations were performed for several different scientific purposes (see Harrison et al. 2013, for a description of the NUSTAR survey programs) and therefore differ significantly in exposure time and signal-to-noise ratio. We use the first three existing NuSTAR observations of NGC 1068, both observations of NGC 1320, and both observations of IC 2560 as these sources are not known to be variable in the hard X-rays. NGC 4945 is known to be variable. We mitigate the effect of the variability by analyzing only one NuSTAR obsID from this source, of which we choose the first of three. We investigate the effect on our conclusions of this choice later in the paper. For a time-resolved analysis of this source, we refer the reader to Puccetti et al. (2014).

For NGC 424, NGC 1320, NGC 1386, NGC 3079, IC 2560, and Mrk 34, we use additional archival data from XMMNewton from 0.5 to $10 \mathrm{keV}$ to provide additional spectral constraints. XMM-Newton data were chosen specifically over other instruments as XMM-Newton and NuSTAR are well matched in terms of effective area and spatial resolution in the energy range where they commonly observe (i.e., $3-10 \mathrm{keV})$. No good-quality data exist for 2MFGC 2280 below $10 \mathrm{keV}$. NGC 1068, NGC 4945, and Circinus are part of a different, non-snapshot $N u S T A R$ program and hence have longer exposure times and higher signal-to-noise spectra and are thus of sufficient quality to constrain the torus parameters well without additional soft X-ray data.

The raw data were reduced using the NUSTARDAS software package version 1.2.1. The events were cleaned and filtered using the nupipeline script with standard parameters. ${ }^{23}$ The nuproducts task was used to generate the spectra and the corresponding response files. Spectra were extracted from circular apertures centered on the peak of the point-source emission, with radii between $30^{\prime \prime}$ and $90^{\prime \prime}$ (larger radii for higher signal-to-background ratio). The background spectra were extracted from regions encompassing the same detector as the source, excluding the source extraction region and avoiding the wings of the PSF as much as possible. Data from both focal plane modules (FPMs) (FPMA and FPMB) and from multiple observations are used for simultaneous fitting, without coadding. Additional details of the data reduction for specific sources can be found in the papers listed above. The observations of NGC 1386 and 2MFGC 2280, which are not published elsewhere, are reduced in a fashion similar to Baloković et al. (2014).

The XMM-Newton data reduction for the observations of NGC 424, NGC 1320, NGC 1386, and NGC 3079 were described in Brightman \& Nandra (2011a), where only EPIC-pn data were used, with source events extracted from $35^{\prime \prime}$ radius circular regions. The events were then filtered for background flares when the level of the background count rate was determined to be twice the level at which the excess variance was determined to be zero. The XMM-Newton data reduction for the observation of Mrk 34 is described in Gandhi et al. (2014), and the XMM-Newton data reduction for IC 2560 is described in Tilak et al. (2008).

All spectra were grouped with a minimum of 20 counts per bin using the HEASARC tool grppha. We use XSPEC version 12.6.0 to carry out X-ray spectral fitting, and the $\chi^{2}$ statistic as the fit statistic, with the background subtracted. For

\footnotetext{
${ }^{23}$ The NuSTAR Data Analysis Software Guide, http://heasarc.gsfc.nasa.gov/ docs/nustar/analysis/NuSTARDAS_swguide_v1.3.pdf.
} 
NUSTAR data, only energies from 3 to $79 \mathrm{keV}$ were considered, as the calibration at lower energies is uncertain, and the NuSTAR response cuts off at $79 \mathrm{keV}$ due to an absorption edge in the optics. XMM-Newton data were used from 1 to $10 \mathrm{keV}$, allowing considerable overlap with NuSTAR.

The primary goal of our analysis is to determine the covering factor of the obscuring material in these CT AGNs using the TORUs model. The AGN emission that is described by this model is by far the dominant emission in the NUSTAR band for these sources; thus, all spectra were fitted with the TORUS model as a baseline. We fix the inclination angle of the torus to the edge-on position of $87^{\circ}$, the maximum inclination angle allowed by the model. As described above, the X-ray spectrum above $10 \mathrm{keV}$ is largely insensitive to the inclination angle, when the inclination angle is greater than $\theta_{\text {tor }}$. Fixing the inclination angle thus reduces the number of free parameters in the fit, and the edge-on choice allows the exploration of the full range of opening angles as opening angles can only be determined up to the inclination angle, after which the source becomes unobscured. Arévalo et al. (2014) find that when fitting the TORUS model to the spectrum of Circinus, both $\theta_{\text {tor }}$ and the inclination angle can be constrained and provide a better fit to the data than if the inclination angle were fixed. We thus allow the inclination angle to be free when fitting the spectrum of Circinus.

While the torus emission is the dominant component in the $N U S T A R$ band, emission not directly associated with the intrinsic AGNs emission or the reprocessing in the torus, such as soft emission from photoionized material, radiative recombination (Guainazzi \& Bianchi 2007), and Thompson-scattered AGNs light, are common in obscured AGNs and are nonnegligible even in the NUSTAR band. Furthermore, due to the size of NuSTAR's PSF, X-ray sources in the host galaxy of the AGNs may also contribute. For example, Puccetti et al. (2014) find that $\sim 60 \%$ of emission in the $4-6 \mathrm{keV}$ band comes from extra-nuclear sources within the NuSTAR extraction region, while Bauer et al. (2014) find that $28 \%$ of the $\mathrm{Fe} \mathrm{K} \alpha$ emission from NGC 1068 comes from the host galaxy. It is expected, however, that these extra-nuclear sources contribute far less at higher energies. We therefore add a power-law model and a thermal plasma component modeled by APEC to broadly account for these soft excess components and extra-nuclear sources. We allow the secondary power-law index to vary freely, likewise for the temperature of the APEC model. For 2MFGC 2280 where only $N U S T A R$ data are available, the soft components were not statistically required, so they were removed from the fit. No secondary power law was required in the fit for IC 2560. For NGC 1068, NGC 4945, and Circinus, where only NuSTAR data were used, the APEC model was not required. This is expected since this model generally describes emission outside the NuSTAR bandpass. The spectra of NGC 424 and NGC 1320 also did not require the APEC model.

NGC 1068 has a well-known Fe complex at 6-7 keV, consisting of neutral $\mathrm{Fe} \mathrm{K} \alpha$ and $\beta$ emission, plus ionized emission at 6.7 and $6.96 \mathrm{keV}$. While the TORUS model accounts for the neutral emission, we add Gaussian components fixed at the energies of the ionized emission to account for these lines, where the widths are fixed at small values $(1 \mathrm{eV})$. As found by Sambruna et al. (2001) and Molendi et al. (2003) for Circinus and Yaqoob (2012) for NGC 4945, we also find that the NUSTAR spectra of these AGNs require an additional Gaussian component each to model lines in the Fe complex, fixed at
Table 2

Details of the Cross-normalizations

\begin{tabular}{|c|c|c|c|}
\hline $\begin{array}{l}\text { Source Name } \\
\text { (1) }\end{array}$ & $\begin{array}{l}\text { FPMB/ } \\
\text { FPMA } \\
(2)\end{array}$ & $\begin{array}{c}X M M-N e w t o n / \\
\text { FPMA } \\
\text { (3) }\end{array}$ & $\begin{array}{l}\text { Models Used } \\
\text { (4) }\end{array}$ \\
\hline NGC 424 & $1.07_{-0.12}^{+0.13}$ & $0.97_{-0.15}^{+0.16}$ & TORUS+POWERLAW \\
\hline NGC 1068 & $1.03_{-0.02}^{+0.03}$ & $\ldots$ & $\begin{array}{l}\text { TORUS+POWERLAW+ZGAUSS } \\
\text { +ZGAUSS }\end{array}$ \\
\hline 2MFGC 2280 & $1.02{ }_{-0.14}^{+0.16}$ & $\cdots$ & TORUS \\
\hline NGC 1320 & $0.91{ }_{-0.07}^{+0.07}$ & $0.89_{-0.12}^{+0.13}$ & TORUS+POWERLAW+ZGAUSS \\
\hline NGC 1386 & $0.80_{-0.15}^{+0.20}$ & $0.84{ }_{-0.16}^{+0.17}$ & $\begin{array}{l}\text { TORUS+POWERLAW+APEC } \\
\quad+\text { ZGAUSS }\end{array}$ \\
\hline NGC 3079 & $1.06_{-0.10}^{+0.11}$ & $0.65_{-0.14}^{+0.16}$ & TORUS+POWERLAW+APEC \\
\hline IC 2560 & $1.07_{-0.09}^{+0.09}$ & $\begin{array}{c}0.822_{-0.11}^{+0.16}, 0.92 \\
{ }_{-0.12}^{+0.17}\end{array}$ & TORUS+APEC+ZGAUSS \\
\hline Mrk 34 & $1.09_{-0.22}^{+0.27}$ & $\begin{array}{c}0.93{ }_{-0.27}^{+0.37}, 0.81 \\
{ }_{-0.24}^{+0.37}\end{array}$ & TORUS+POWERLAW+APEC \\
\hline NGC 4945 & $1.02_{-0.02}^{+0.02}$ & $\cdots$ & TORUS+POWERLAW+ZGAUSS \\
\hline Circinus & $1.03_{-0.01}^{+0.01}$ & $\cdots$ & TORUS+POWERLAW+ZGAUSS \\
\hline
\end{tabular}

Note. Column (2) gives the cross-normalization between the two FPMs, column (3) gives the cross-normalization between FMPA and XMMNewton (MOS 1 and MOS 2, respectively, for IC 2560 and pn and combined MOS for Mrk 34), and column (4) lists the models used to fit each of the spectra.

$6.7 \mathrm{keV}$ in NGC 4945 and allowed to vary around $6.6 \mathrm{keV}$ for Circinus, as required by the data. Additional Gaussian components at the energies of the Fe complex were required to fit the spectra of NGC 1320, NGC 1386, and IC 2560. We summarize all the models used for each source in Table 2.

We allow the cross-normalization between the two $\mathrm{NuS}$ $T A R$ FPMs to vary in the fitting to account for instrumental cross-calibration. We also allow the cross-normalization between the FPMs and XMM-Newton to vary to account for instrumental cross-calibration and the fact that the $X M M$ Newton observations are not simultaneous with NuSTAR. We fix the other parameters to each other since there is a known agreement between spectral parameters determined from XMMNewton and NuSTAR (e.g., Walton et al. 2013, 2014). We present the cross-normalizations in Table 2. The observations are mainly consistent with the cross-normalization between the FPMs being unity, with the exceptions being NGC 1068, NGC 1320, and Circinus. NGC 1068 and Circinus both show the normalization of FPMB to be $3 \%$ greater than the normalization of the FPMA. For NGC 1320 the FPMB normalization is 9\% lower. Baloković et al. (2014) also investigate the cross-normalization between the two FPMs for NGC 424, NGC 1320, and IC 2560, and our results agree. Full details of NuSTAR in-orbit calibration are presented in $\mathrm{K} . \mathrm{K}$. Madsen et al. (2015, in preparation).

\section{SPECTRAL FITTING RESULTS}

Our spectral fitting with the TORUS model reproduces the data well in all 10 sources, with reasonable reduced $\chi^{2}$. We confirm the CT nature of all $10 \mathrm{AGNs}$, with $N_{\mathrm{H}}$ constraints in excess of $1.5 \times 10^{24} \mathrm{~cm}^{-2}$. The CT nature of 2MFGC 2280 is shown here for the first time.

The best-fit spectral parameters are presented in Table 3, along with their $90 \%$ confidence intervals calculated using a $\Delta \chi^{2}=4.61$ criterion on two interesting parameters in order to 
Table 3

Best-fit Spectral Parameters of the TORUS and SPHERE Models

\begin{tabular}{|c|c|c|c|c|c|c|c|c|c|}
\hline \multicolumn{10}{|c|}{ TORUS Fits } \\
\hline $\begin{array}{l}\text { Source Name } \\
\text { (1) }\end{array}$ & $\begin{array}{l}\text { Redshift } \\
\text { (2) }\end{array}$ & $\begin{array}{c}\text { PHA Bins } \\
\text { (3) }\end{array}$ & $\begin{array}{l}\chi^{2} \\
(4)\end{array}$ & $\begin{array}{l}\chi_{r}^{2} \\
(5)\end{array}$ & $\begin{array}{l}N_{\mathrm{H}} \\
(6)\end{array}$ & $\begin{array}{l}\Gamma \\
(7)\end{array}$ & $\begin{array}{l}\theta_{\text {tor }} \\
(8)\end{array}$ & $\begin{array}{c}\log _{10} F_{X} \\
(9)\end{array}$ & $\begin{array}{c}\log _{10} L_{X} \\
(10)\end{array}$ \\
\hline NGC 1068 & 0.0038 & 1140 & 1950.47 & 1.72 & $6.32_{-0.05}^{+0.44}$ & $2.31{ }_{-0.05}^{+0.04}$ & $58.99_{-2.76}^{+4.51}$ & -10.51 & $42.87_{-0.06}^{+0.04}$ \\
\hline 2MFGC 2280 & 0.0150 & 36 & 13.18 & 0.43 & $2.50_{-1.00}^{+0.58}$ & $1.966_{-0.56}^{+0.26}$ & $78.97{ }_{-41.87}^{+5.03}$ & -10.98 & $43.26_{-0.74}^{+0.31}$ \\
\hline NGC 1320 & 0.0089 & 172 & 190.37 & 1.18 & $100.00_{-70.81}^{+u}$ & $1.66_{-0.19}^{+0.21}$ & $60.17_{-14.91}^{+10.69}$ & -10.95 & $42.79{ }_{-0.09}^{+0.12}$ \\
\hline IC 2560 & 0.0096 & 165 & 182.51 & 1.19 & $100.00{ }_{-86.64}^{+u}$ & $2.53{ }_{-0.20}^{+0.20}$ & $59.08_{-20.05}^{+9.57}$ & -11.66 & $42.95_{-0.13}^{+0.11}$ \\
\hline Mrk 34 & 0.0510 & 69 & 71.03 & 1.22 & $50.43_{-31.01}^{+49.57}$ & $1.73_{-0.56}^{+1.25}$ & $72.51{ }_{-46.51}^{+6.89}$ & -11.52 & $44.18{ }_{-0.38}^{+0.39}$ \\
\hline NGC 4945 & 0.0019 & 906 & 1108.75 & 1.24 & $2.54_{-0.15}^{+0.13}$ & $1.58{ }_{-0.04}^{+0.04}$ & $26.00_{-l}^{+0.50}$ & -9.65 & $41.92{ }_{-0.08}^{+0.07}$ \\
\hline Circinus & 0.0014 & 1715 & 1880.27 & 1.10 & $4.85_{-0.42}^{+0.39}$ & $2.27_{-0.07}^{+0.05}$ & $33.79_{-1.56}^{+1.83}$ & -9.63 & $42.51_{-0.09}^{+0.07}$ \\
\hline & & & & & ERE Fits & & & & \\
\hline NGC 1386 & 0.0029 & 71 & 73.74 & 1.25 & $0.76_{-0.19}^{+0.45}$ & $3.00{ }_{-l}^{+u}$ & $10.00_{-5.44}^{+u}$ & -11.56 & $39.67{ }_{-0.11}^{+0.18}$ \\
\hline NGC 3079 & 0.0037 & 214 & 266.42 & 1.31 & $1.84_{-0.32}^{+0.32}$ & $1.86_{-0.23}^{+0.30}$ & $1.42_{-0.43}^{+0.34}$ & -10.83 & $41.53_{-0.43}^{+0.45}$ \\
\hline NGC 4945 & 0.0019 & 905 & 972.64 & 1.09 & $2.25_{-0.07}^{+0.24}$ & $1.78_{-0.06}^{+0.13}$ & $1.38_{-0.37}^{+0.16}$ & -9.66 & $42.05_{-0.10}^{+0.17}$ \\
\hline
\end{tabular}

Note. Parameters determined by the ToRus model are listed in the top rows. For NGC 1386, IC 3079, and NGC 4945 we also present the best-fit parameters from the SPHERE model as the TORUS model finds that these sources are highly covered (bottom rows). Column (1) lists the source name, column (2) gives the redshift of the source, column (3) shows the total number of pulse height analysis (PHA) bins used in the spectrum, column (4) gives the $\chi^{2}$ of the fit, column (5) gives the reduced $\chi^{2}$, equal to $\chi^{2}$ divided by the number of degrees of freedom in the fit, column (6) gives the $N_{\mathrm{H}}$ in units of $10^{24} \mathrm{~cm}^{-2}$, with uncertainties, column (7) gives the photonindex, column (8) gives $\theta_{\text {tor }}$ determined by the TORUs model in degrees or the iron abundance with respect to solar hydrogen abundance from the SPHERE model, column (9) gives the logarithm of the observed 3-79 keV NuSTAR flux in $\mathrm{erg} \mathrm{cm}^{-2} \mathrm{~s}^{-1}$, and column (10) gives the logarithm of the intrinsic (deabsorbed) rest-frame $2-10 \mathrm{keV}$ luminosity in $\mathrm{erg} \mathrm{s}^{-1} .+u$ indicates that a parameter has reached the upper limit when estimating the uncertainty. $-l$ indicates that the lower limit has been reached.

Table 4

Best-fit Spectral Parameters of the Secondary Power Law, APEC, and Gaussian Line Models

\begin{tabular}{|c|c|c|c|c|c|c|}
\hline $\begin{array}{l}\text { Source Name } \\
\text { (1) }\end{array}$ & $\begin{array}{c}\Gamma \\
(2)\end{array}$ & $\begin{array}{c}A_{\mathrm{pl}} / 10^{-5} \\
(3)\end{array}$ & $\begin{array}{l}T_{\text {apec }} \\
(4)\end{array}$ & $\begin{array}{c}A_{\text {apec }} / 10^{-5} \\
(5)\end{array}$ & $\begin{array}{c}E_{\text {line }} \\
(6)\end{array}$ & $\begin{array}{c}A_{\text {line }} / 10^{-5} \\
(7)\end{array}$ \\
\hline \multicolumn{7}{|c|}{ TORUS Fits } \\
\hline NGC 1068 & $2.29{ }_{-0.09}^{+0.10}$ & $163.20_{-21.06}^{+19.07}$ & $\ldots$ & $\ldots$ & $\ldots$ & $\ldots$ \\
\hline 2MFGC 2280 & $\ldots$ & $\ldots$ & $\ldots$ & $\ldots$ & $\ldots$ & $\ldots$ \\
\hline NGC 1320 & $3.70{ }_{-0.41}^{+0.46}$ & $12.91_{-2.51}^{+3.11}$ & $\ldots$ & $\ldots$ & $6.55_{-0.04}^{+0.05}$ & $0.57{ }_{-0.17}^{+0.20}$ \\
\hline IC 2560 & $\ldots$ & $\ldots$ & $0.68_{-0.21}^{+0.20}$ & $2.28{ }_{-0.62}^{+1.16}$ & $6.43{ }_{-0.01}^{+0.02}$ & $0.677_{-0.15}^{+0.16}$ \\
\hline Mrk 34 & $2.90_{-0.28}^{+0.81}$ & $1.98_{-1.19}^{+0.99}$ & $0.82_{-0.05}^{+0.38}$ & $0.92_{-0.54}^{+0.49}$ & $\ldots$ & $\ldots$ \\
\hline NGC 4945 & $1.900_{-0.48}^{+0.55}$ & $29.72_{-16.28}^{+38.76}$ & $\ldots$ & $\ldots$ & $\ldots$ & $\ldots$ \\
\hline Circinus & $2.05_{-1.19}^{+1.96}$ & $226.28_{-226.28}^{+1698.37}$ & $\cdots$ & $\cdots$ & $6.57_{-0.00}^{+0.04}$ & $12.86_{-1.12}^{+1.17}$ \\
\hline NGC 4945 & $1.00_{-0.25}^{+0.22}$ & $12.57_{-4.41}^{+5.80}$ & $\ldots$ & $\ldots$ & $\cdots$ & $\ldots$ \\
\hline
\end{tabular}

Note. Column (1) gives the source name, column (2) gives the power-law index of the secondary power law, column (3) gives the normalization of the secondary power law, column (4) gives the temperature of the APEC model in keV, column (5) gives the normalization of the APEC model, column (6) gives the energy of the Gaussian line in $\mathrm{keV}$, and column (7) gives the normalization of the Gaussian line. 


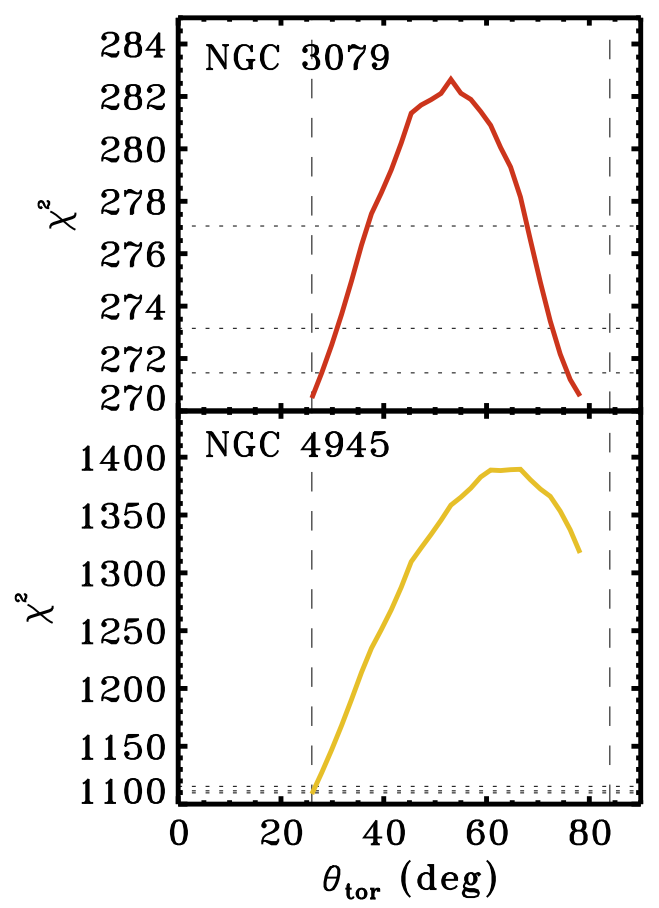

Figure 5. $\chi^{2}$ as a function of $\theta_{\text {tor }}$ for fits to NGC 3079 and NGC 4945, showing local minima at both small and large opening angles. For NGC 3079 the large opening angle is marginally favored, while in NGC 4945 the small opening angle is significantly favored. The dashed lines indicate the minimum $\left(26^{\circ}\right)$ and maximum $\left(84^{\circ}\right) \theta_{\text {tor }}$ values allowed by the model, and the dotted lines show the $68 \%, 90 \%$, and $99 \%$ confidence levels, which correspond to $\Delta \chi^{2}$ values of $1.00,2.71$, and 6.63 , respectively.

minimize degeneracies. The uncertainty on the luminosity is propagated from the uncertainty on the normalization of the TORUS model. Details of the parameters of the components used to fit the soft X-ray excesses are listed in Table 4.

The best-fit $\theta_{\text {tor }}$ measured in our sample spans a large range, from $26^{\circ}$ to $80^{\circ}$, limited by the allowed range of the model. The uncertainties on $\theta_{\text {tor }}$ range from $5^{\circ}$ to $40^{\circ}$, with the best constraints coming from NGC 1068, NGC 4945, and Circinus, which have the highest signal-to-noise spectra in the sample. While the fit to Mrk 34 gives a fairly large $\theta_{\text {tor }}$, the constraints are poor, with statistically allowed values ranging from $26^{\circ}$ to $80^{\circ}$. The case is similar for 2 MFGC 2280 , albeit with slightly tighter constraints.

In two sources, NGC 3079 and NGC 4945, we discover bimodality in statistic space for $\theta_{\text {tor }}$, with local minima at small and large opening angles. We show their $\chi^{2}$ as a function of $\theta_{\text {tor }}$ in Figure 5. For NGC 3079, the large opening angle provides a marginally better fit, whereas for NGC 4945 the small opening angle is a significantly better fit.

For two sources, NGC 1386 and NGC 4945, we find that the best-fit $\theta_{\text {tor }}$ reaches the lower limit on this parameter $\left(26^{\circ}\right)$, which implies that both the sources are highly covered. We also find that the $\Gamma$ values for these fits deviate significantly from the canonical value of 1.9 (steep at 2.52 for NGC 1386 and flat for NGC 4945 at 1.58). The fact that the deviations from the canonical value occur at the edge of the $\theta_{\text {tor }}$ parameter space suggests that the true $\theta_{\text {tor }}$ of these sources lies outside of the range of the TORUs model (i.e., $\theta_{\text {tor }}<26^{\circ}$ ).

Brightman \& Nandra (2011a) also present a model of a fully covered source (i.e., $0^{\circ}$ opening angle), which includes variable iron and elemental abundances, known as SPHERE. We fit this model, with the iron abundance free, in place of the TORUS model for NGC 1386 and NGC 4945, and present the results in Table 3. This is also done for NGC 3079 since the small opening angle also provides a good fit to that source. An improvement in the fit statistic is found for both NGC 3079 and NGC 4945, where the spherical model produces a significantly better ( $>90 \%$ significance) fit than the TORUS model for both $\left(\Delta \chi^{2}=4\right.$ and 136, respectively). The iron abundance has been estimated to be slightly higher, albeit statistically consistent with solar metalicity. The $\Gamma$ inferred from the SPHERE model is also consistent with the canonical value for both sources. For NGC 1386, the SPHERE model produces extreme $\Gamma$ and iron abundances values and; therefore, it is not likely to be a good description of the spectrum.

Due to the range of $\theta_{\text {tor }}$ allowed for the ToRus model, we cannot test if a very large opening angle $\left(\sim 90^{\circ}\right)$ would provide as equally good a solution for NGC 3079 or NGC 4945. However, for the local minimum at the largest angle, the $\Gamma$ for NGC 4945 is even flatter (1.43) than the smallest opening angle and does not suggest that a large opening angle would describe the spectrum well. For this solution, $N_{\mathrm{H}}=2.64 \times 10^{24}$ $\mathrm{cm}^{-2}$ and $L_{\mathrm{X}}=2.27 \times 10^{42} \mathrm{erg} \mathrm{s}^{-1}$.

We conclude from this that NGC 3079 and NGC 4945 are heavily buried sources with a covering factor close to unity. For all further analysis we assign a value of $\theta_{\text {tor }}=0^{\circ}$ to these sources. We discuss this result later, especially for NGC 4945, where previous results have favored a very low covering factor (e.g., Madejski et al. 2000).

The best-fit unfolded spectra fits with the TORUs model are presented in Figure 6. This illustrates the variety of spectral shapes seen above $10 \mathrm{keV}$ for these CT sources, as well as the range in signal-to-noise ratio in the sample. The data-to-model ratios are presented in Figure 7, which indicate how well the TORUS model fits the data. The worst reduced $\chi^{2}$ from the TORUS fits result from the spectrum of NGC 1068 (1.72), where there is significant curvature in the data-to-model ratio, implying that the TORUS model is not a good description of the data. This was also the conclusion from the detailed analysis of this source in Bauer et al. (2014), which utilizes data from a large array of telescopes and several model combinations. They find that the data require a complex combination of models to reduce this curvature and that a monolithic torus structure is not likely.

First, in our investigation into what drives the covering factor in AGNs, we examine how the measurement of $\theta_{\text {tor }}$ in our analysis relates to the other measurements made with the TORUs model, namely, $N_{\mathrm{H}}$ and $\Gamma$, by plotting these quantities against each other along with their uncertainties (Figure 8). No uncertainties can be derived for the $\theta_{\text {tor }}=0^{\circ}$ values assigned to NGC 3079 and NGC 4945 as these have been determined from the SPHERE model, which has a fixed opening angle of zero. There is no clear relationship between these quantities.

\section{POTENTIAL BIASES AND SYSTEMATICS}

In our analysis, we fix the torus inclination angle to an edgeon position of $87^{\circ}$. This is primarily motivated by the observation that the inclination angle does not have a large effect on the observed spectrum above $10 \mathrm{keV}$ and thus allows us to reduce the number of free parameters in the fit. Furthermore, an edge-on inclination angle allows the full range of opening angles to be explored. Fixing the inclination angle also avoids the scenario where the inclination angle approaches the opening angle, which produces a partially covered solution 


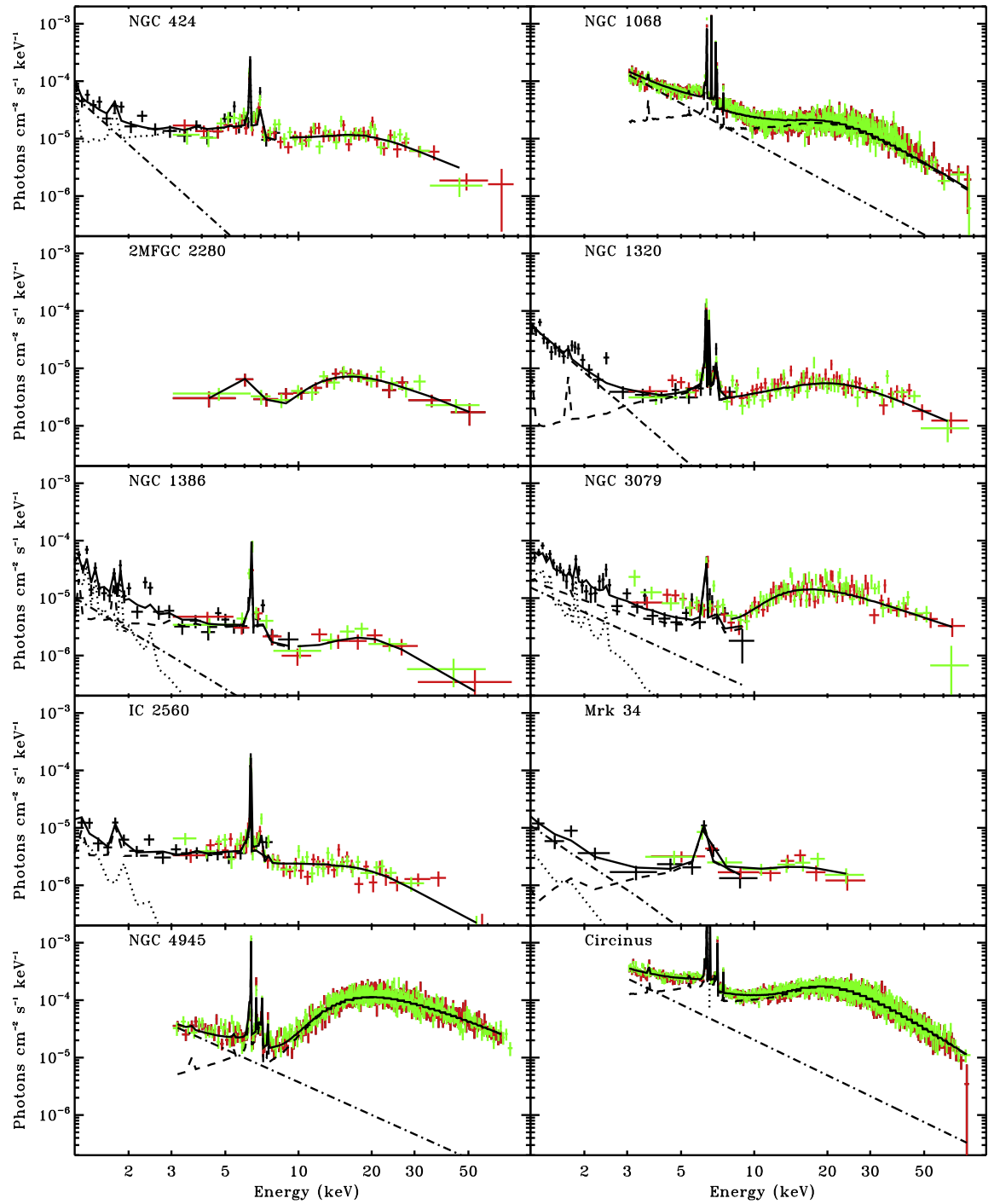

Figure 6. Unfolded spectra for all sources fit with the TORUs model, where NuSTAR FPMA data are shown in red, FPMB data are shown in green, and XMMNewton data are shown in black. The solid black lines represent the sum of all model components, while the dashed line shows the ToRus model. Dotted-dashed lines represent the secondary power-law model, and the dotted lines represent the thermal plasma model APEC, both used to fit the soft-X-rays. The spectra have been binned for plotting purposes, so that each bin has a detection of at least $3 \sigma$ up to a maximum of 10 data points per bin.

due to the angular binning in the TORus model. We investigate whether allowing the inclination angle to be free allows it to be constrained. This is not the case, but Circinus is an exception, as found by Arévalo et al. (2014); thus, we have allowed the inclination angle to be free for this source.

The NUSTAR band is also well suited to studying the highenergy cutoff in the X-ray spectra of AGNs and has been used to constrain this in a number of bright unobscured sources (e.g., SWIFT J2127.4+5654, IC 4329 A, 3C 382, MCG-5-2316; Ballantyne et al. 2014; Brenneman et al. 2014; Marinucci et al. 2014; Baloković et al. 2015, respectively). However, the TORUs model does not include this parameter, and thus we have neglected its effect in this work. The potential effect of neglecting the high-energy cutoff here is to overestimate the Compton hump, which is produced by the down-scattering of high-energy photons. As the covering factor roughly correlates with the strength of the Compton hump, then overestimating the Compton hump will lead to a systematic underestimation of the covering factor. We expect this effect to be very small as the lowest high-energy cutoff energy detected so far by
NuSTAR is at $108 \mathrm{keV}$ in SWIFT J2127.4+5654 (Marinucci et al. 2014), which is far above that of the Compton hump $(\sim 30 \mathrm{keV})$. For photons originating around a cutoff of this energy to affect the Compton hump, at least 10 scatterings would be required. To understand this effect fully, the TORUS model should be improved to include the high-energy cutoff.

For this study, we have a heterogeneous sample in terms of $\mathrm{X}$-ray spectral coverage, and most of our sample has accompanying soft X-ray data to constrain the spectral components below $10 \mathrm{keV}$. However, for four sources, we use NUSTAR data alone. No soft X-ray data exist for 2MFGC 2280; meanwhile, for NGC 1068, NGC 4945, and Circinus, the NUSTAR data have high enough signal-to-noise ratio that they can constrain the TORUS parameters alone. We test this assumption for all three sources by adding in an $X M M$ Newton observation to model the soft X-rays below $3 \mathrm{keV}$. We find that an additional APEC model is required to fit these data in addition to the TORUs and power-law model already in place. Fitting the data simultaneously with the soft component constrained by XMM-Newton produces only small changes in 


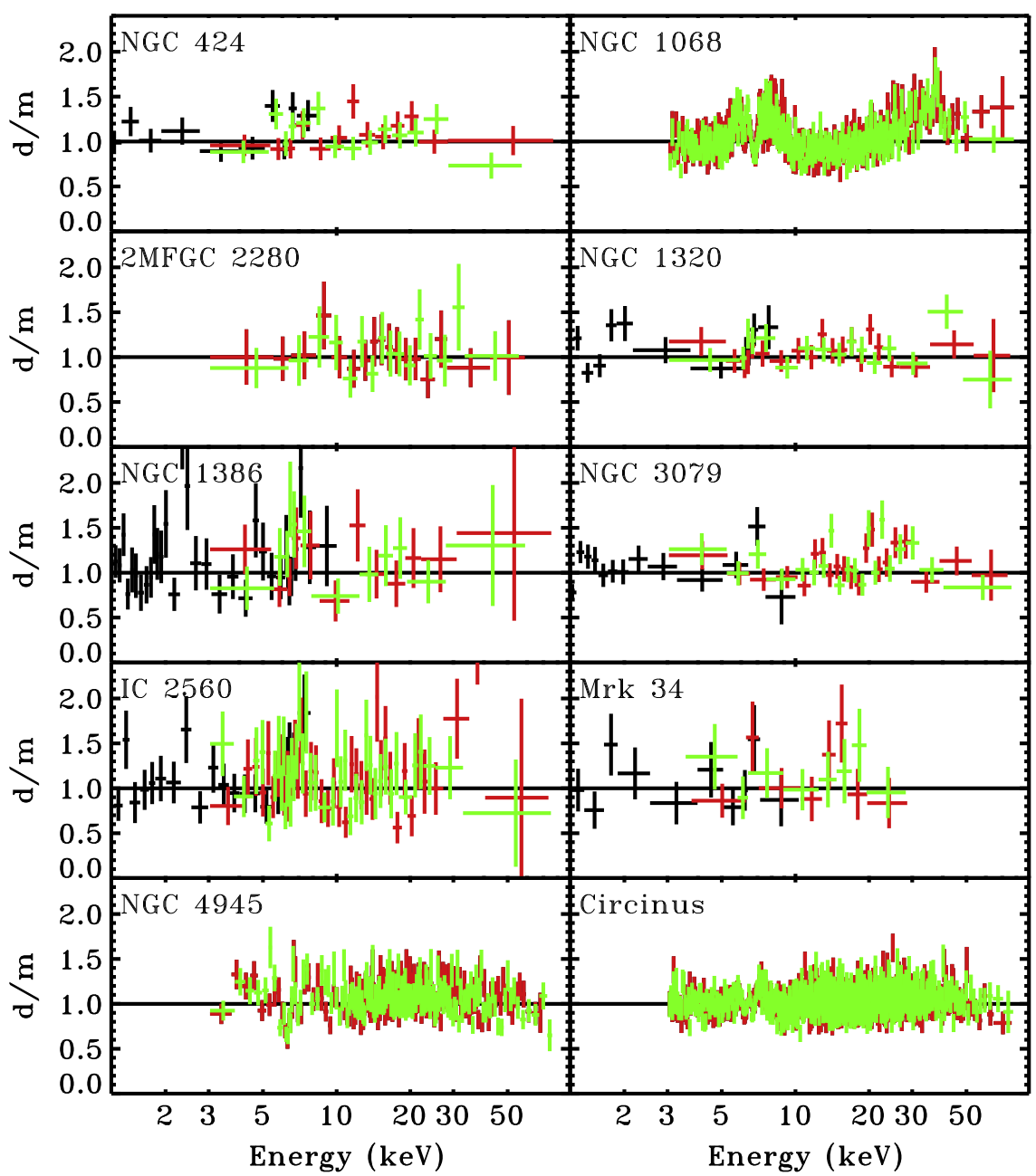

Figure 7. Data-to-model ratios of the fits with the ToRus model. The colors are the same as Figure 5. The binning for this figure has been increased in order to better see deviations and trends in the residuals.

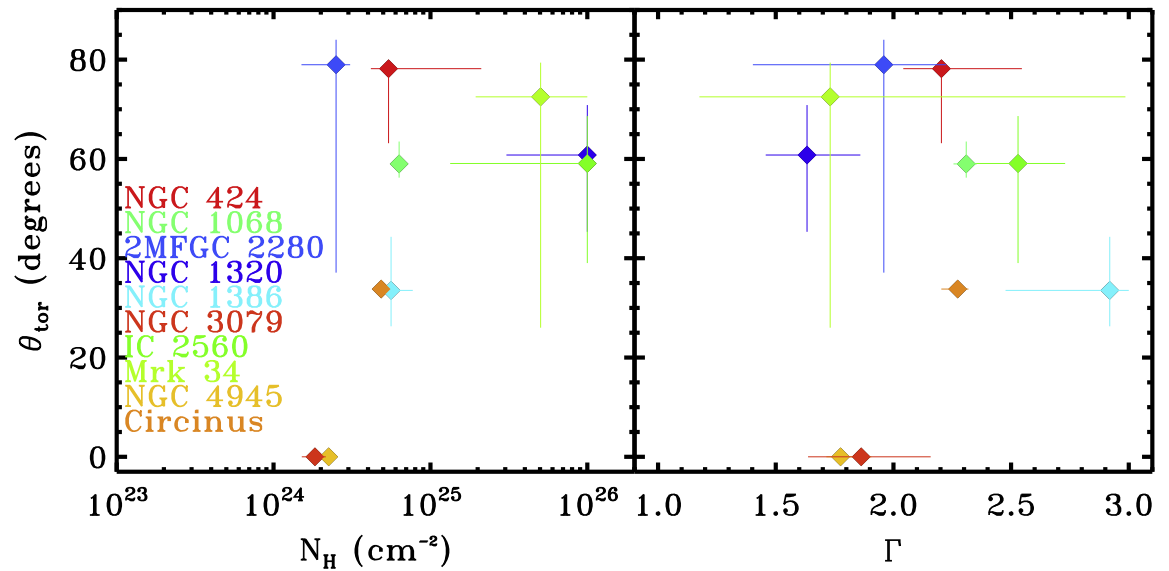

Figure 8. Best-fit measurements of $\theta_{\text {tor }}$ against best-fit measurements of $N_{\mathrm{H}}$ and $\Gamma$ from the TORus model. NGC 3079 and NGC 4945 are best fit by the SPHERE model, implying that these sources have $\theta_{\text {tor }}=0^{\circ}$. No uncertainties can be calculated for this, however, as the SPHERE model has a fixed opening angle of zero.

$\theta_{\text {tor }}$, and within measurement uncertainties. We conclude that the exclusion of the soft X-ray data does not bias our results significantly in these sources.

When fitting XMM-Newton and NuSTAR data together, we have kept the spectral parameters fixed between the two data sets. Since the observations were not made simultaneously, spectral variability could be missed, with the exception of the intrinsic luminosities, which is accounted for by the crossnormalization between the two observatories being a free parameter. We investigated spectral variability in the five sources where we use both XMM-Newton and NuSTAR data together by allowing the $N_{\mathrm{H}}$ parameter to vary between data sets. We find that the $N_{\mathrm{H}}$ parameter is consistent within the uncertainties for all of these except NGC 424. For this source, 
Table 5

Comparison of Fits with the torus Model to Previous Analyses with PEXRAV and MYTORUs

\begin{tabular}{|c|c|c|c|c|c|c|}
\hline $\begin{array}{l}\text { Source Name } \\
\text { (1) }\end{array}$ & $\begin{array}{l}N_{\mathrm{H}} \text { (MYTORUS) } \\
(2)\end{array}$ & $\begin{array}{c}N_{\mathrm{H}} \text { (TORUS) } \\
(3)\end{array}$ & $\begin{array}{c}\Gamma \text { (PEXRAV) } \\
(4)\end{array}$ & $\begin{array}{c}\Gamma \text { (MYTORUS) } \\
(5)\end{array}$ & $\begin{array}{c}\Gamma \text { (TORUS) } \\
(6)\end{array}$ & $\begin{array}{l}\theta_{\text {tor }} \\
(7)\end{array}$ \\
\hline NGC 424 & $3 \pm 1$ & $5.41_{-1.23}^{+15.67}$ & $1.66 \pm 0.09$ & $2.07{ }_{-0.09}^{+0.11}$ & $2.20_{-0.16}^{+0.34}$ & $78.19_{-15.01}^{+0.43}$ \\
\hline NGC 1068 & $10_{-4.4}^{+u}$ & $6.32_{-0.05}^{+0.44}$ & $1.57 \pm 0.02$ & $2.20_{-0.01}^{+0.02}$ & $2.31_{-0.05}^{+0.04}$ & $58.99_{-2.76}^{+4.51}$ \\
\hline NGC 1320 & $4_{-2}^{+4}$ & $100.00_{-70.81}^{+u}$ & $1.3 \pm 0.1$ & $1.6 \pm 0.2$ & $1.66_{-0.19}^{+0.21}$ & $60.17_{-14.91}^{+10.69}$ \\
\hline IC 2560 & $10_{-3}^{+u}$ & $100.00_{-86.64}^{+u}$ & $2.2_{-0.2}^{+0.1}$ & $2.55(\mathrm{f})$ & $2.53{ }_{-0.20}^{+0.20}$ & $59.08_{-20.05}^{+9.57}$ \\
\hline Mrk 34 & $2.45_{-1.08}^{+u}$ & $50.43_{-31.01}^{+49.57}$ & $\ldots$ & $2.2_{-0.3}^{+0.2}$ & $1.73_{-0.56}^{+1.25}$ & $72.51_{-46.51}^{+6.89}$ \\
\hline
\end{tabular}

Note. From Baloković et al. (2014) for NGC 424, NGC 1320, and IC 2560, Bauer et al. (2014) for NGC 1068, Gandhi et al. (2014) for Mrk 34, Puccetti et al. (2014) for NGC 4945, and Arévalo et al. (2014) for Circinus. Column (1) lists the source name, column (2) gives the $N_{\mathrm{H}}$ measured by MYTORUs in units of $10^{24} \mathrm{~cm}^{-2} .+u$ indicates that the upper constraint on the $N_{\mathrm{H}}$ from mYTORUs is beyond the upper limit of the model. Column (3) gives the $N_{\mathrm{H}}$ measured by the toRUs model in the same units, column (4) gives the photon index measured by PEXRAV, column (5) gives the photon index measured by MYTORUS, where (f) indicates that this parameter has been fixed, and column (6) gives the photon index measured by ToRus. Column (7) lists $\theta_{\text {tor }}$ measured by the ToRus model in units of degrees. $+u$ indicates that a parameter has reached the upper limit when estimating the uncertainty. $-l$ indicates that the lower limit has been reached.

XMM-Newton measures $N_{\mathrm{H}}=4.6_{-0.7}^{+1.3} \times 10^{24} \mathrm{~cm}^{-2}$ and $N u S$ TAR measures $N_{\mathrm{H}}=2.1_{-0.3}^{+0.6} \times 10^{24} \mathrm{~cm}^{-2}$. Allowing for the $N_{\mathrm{H}}$ to vary like this does not, however, change our result on $\theta_{\text {tor }}$.

Lastly, we investigate how further spectral components added to the fit may affect our results. Specifically, we investigate the addition of the semi-infinite slab reflection model PEXRAV in order to represent a contribution to reflection from the accretion disk. We use the pure reflection component of this model and fix $\Gamma$ to that of the TORUs model and set the high-energy cutoff to maximum $\left(1 \times 10^{6} \mathrm{eV}\right)$, the adundances to solar, and the cosine of the inclination angle to 0.45 . The normalization is free. In five sources, NGC 1068, 2MFGC 2280, NGC 1320, NGC 1386, and IC 2560, the normalization of the PEXRAV component falls to zero in the fit. For NGC 3079 and NGC 4945, the addition of the PEXRAV component has no effect on $\theta_{\text {tor }}$, whereas for Circinus, the change is $-5^{\circ}$. For NGC 424 the addition of PEXRAV to the fit causes a shift in $\theta_{\text {tor }}$ of $-25^{\circ}$. While the change in $\theta_{\text {tor }}$ is large for NGC 424, when considering the sample as a whole we conclude that the addition of this component does not appear to introduce a systematic effect in the determination of $\theta_{\text {tor }}$. We note that the PEXRAV component added here is unabsorbed and not subjected to the same absorption as the primary power law. To treat this correctly, the PEXRAV spectrum would need to be added to the intrinsic emission of the Monte Carlo models that calculate the effect of Compton scattering within the TORUS model, which is not easily done.

\section{COMPARISON TO PREVIOUS RESULTS}

The use of the TORUs model here to determine the opening angle of the torus is fairly novel, as is the use of X-ray torus models to understand high-energy emission in heavily obscured AGNs. Therefore, in Section 2 we compared the spectral fit parameters determined from the classical PEXRAV model and the more recent MYTORUS model when fitted to simulated spectra from the TORUS model for a range of $\theta_{\text {tor }}$ and $N_{\mathrm{H}}$. Here, we compare the fits to real NUSTAR data with the TORUS model presented here to those made with PEXRAV and MYTORUS from previous published works. We list the different $N_{\mathrm{H}}$ and $\Gamma$ values obtained from each in Table 5.

In our initial analysis, we found that PEXRAV systematically underestimates $\Gamma$ with respect to both TORUS and MYTORUS, most likely because it cannot reproduce the full strength of the Compton hump produced by a torus geometry. This is also found in our comparison of fits to real data, as fits with PEXRAV produce a lower $\Gamma$ than both the TORUS or MYTORUS model for NGC 424, NGC 1068, NGC 1320, and IC 2560.

As for fits with MYTORUS with respect to the TORUS model, the $\Gamma$ measurements are consistent with each other within the uncertainties, with the exceptions of NGC 1068 and NGC 4945. For NGC 1068, Bauer et al. (2014) use a large, combined set of X-ray spectral data from different observatories with far more detailed spectral modeling than is done here, including modeling of the host spectrum. Futhermore, the MYTORUS fits are done in decoupled mode. For NGC 4945, the TORUS model measures $\Gamma \sim 0.2$ lower than MYTORUS. However, the measurement of $\Gamma$ using MYTORUs in Puccetti et al. (2014) was done on a time-resolved basis with MYTORUs in decoupled mode, where the parameters of the reflection components are not fixed to those of the transmitted component. However, we do measure a lower $N_{\mathrm{H}}$ for NGC 4945 than the MYTORUS model, which may be the reason for the discrepancy. Furthermore, a fit with the SPHERE model produces a better fit to the data and a $\Gamma$ value consistent with MYTORUS $\left(1.78_{-0.06}^{+0.13}\right)$. For Mrk 34 , the difference in the measured $\Gamma$ values between the two models is $\sim 0.5$, with MYTORUS producing the steeper slope. The TORUS model, however, measures $N_{\mathrm{H}}>10^{25} \mathrm{~cm}^{-2}$ in Mrk 34, whereas MYTORUS is limited to $N_{\mathrm{H}}<10^{25} \mathrm{~cm}^{-2}$, and indeed the upper limit is reached in the fit. The inability of MYTORUs to measure columns greater than this is the likely cause of disagreement in Mrk 34, as also noted by Gandhi et al. (2014). The two measured values are nevertheless consistent within the large uncertainties in $\Gamma$.

Comparing our derived covering factors to those determined at other wavelengths would also be insightful. Recently, Ichikawa et al. (2015) used infrared clumpy torus models and measured covering factors from these for two of our sample, NGC 1386 and Circinus, finding $0.87_{-0.11}^{+0.05}$ and $0.96_{-0.03}^{+0.02}$, respectively. These values compare favorably with our measurements of $0.83_{-0.12}^{+0.06}$ and $0.83_{-0.02}^{+0.01}$.

\section{1. $N G C 4945$}

The high covering factor determined here for NGC 4945 is in disagreement with previous analyses that conclude that 
NGC 4945 has a small covering factor, of order 0.1 (Madejski et al. 2000; Done et al. 2003; Yaqoob 2012; Puccetti et al. 2014). This conclusion was drawn from the fact that NGC 4945 is variable above $10 \mathrm{keV}$ and that it has a relatively weak reflected component. Madejski et al. (2000) used Monte Carlo simulations to show that the fraction of unscattered photons reaching the observer is much higher for low covering factors $\left(63 \%\right.$ for $\left.\theta_{\text {tor }}=80^{\circ}\right)$ than high covering factors $(19 \%$ for $\left.\theta_{\text {tor }}=10^{\circ}\right)$. The argument states that with an optical depth to Compton scattering of $2-3$, the intrinsic variability of the AGNs with a high covering factor would be smeared out by reflection and that a low covering factor would be required to produce such weak reflection.

Done et al. (2003) also conclude that the CT material cannot cover the whole source in NGC 4945 as the $6.4 \mathrm{keV}$ emission is spatially extended in Chandra observations and must be illuminated by hard X-rays from the AGNs. Done et al. (2003) also suggest that NGC 4945 must be fully covered due to the lack of high-ionization optical/IR lines, although this material does not need to be CT.

Yaqoob (2012) presented an extensive broadband X-ray spectral analysis of NGC 4945 using Suzaku, BeppoSAX, and Swift/BAT data using MYTORUS, TORUS, and SPHERE models and also prefer the small covering factor solution due to the hard Xray variability.

A detailed spectral and temporal analysis of NGC 4945 with NuSTAR was presented in Puccetti et al. (2014) utilizing the MYTORUS model in decoupled mode. The hard X-ray variability was confirmed, where variations of a factor of two above $10 \mathrm{keV}$ were reported. They estimate the covering factor for this source, using the ratio of the reflected component to the direct component. Since this is very small, they conclude that the covering factor is $\sim 0.13$ and fairly constant with flux.

Using the TORUS model, we have found that the X-ray spectral shape of NGC 4945 can also be produced by a source with a high covering factor of obscuring CT material and that for this model this in fact provides a better fit to the X-ray spectrum than the low covering factor scenario. Figure 5 shows $\chi^{2}$ as a function of $\theta_{\text {tor }}$ for the fit to NGC 4945. Although this shows a local minimum at large opening angles (low covering factor), the minimum at small opening angles (large covering factor) is significantly lower. Indeed, with their Monte Carlo simulations, Madejski et al. (2000), using RXTE data, also find that the spectral shape favors a small opening angle, finding $\chi^{2}=68.5$ for $\theta_{\text {tor }}=20^{\circ}$ and $\chi^{2}=75.4$ for $\theta_{\text {tor }}=80^{\circ}$. Their conclusion regarding the large opening angle is instead driven by the variability and their Monte Carlo simulations. However, it is not clear whether these results based on the variability are dependent on the geometry of the torus used (a torus with a square cross section), or if these results are energy dependent.

Our results also imply an optical depth to scattering of $1.5-1.7$, which is lower than previously considered, and thus the effect of scattering is slightly diminished. However, the fraction of directly transmitted photons is still low when considering a high covering factor, which is hard to reconcile with the hard X-ray variability.

The conclusions regarding the covering factor of CT material surrounding NGC 4945 drawn from the variability and those drawn from the spectral shape are at odds, and the models used to draw these conclusions both have their limitations. Concerning the variability, it has been assumed that scattered photons cannot transmit the intrinsic variability of the source.

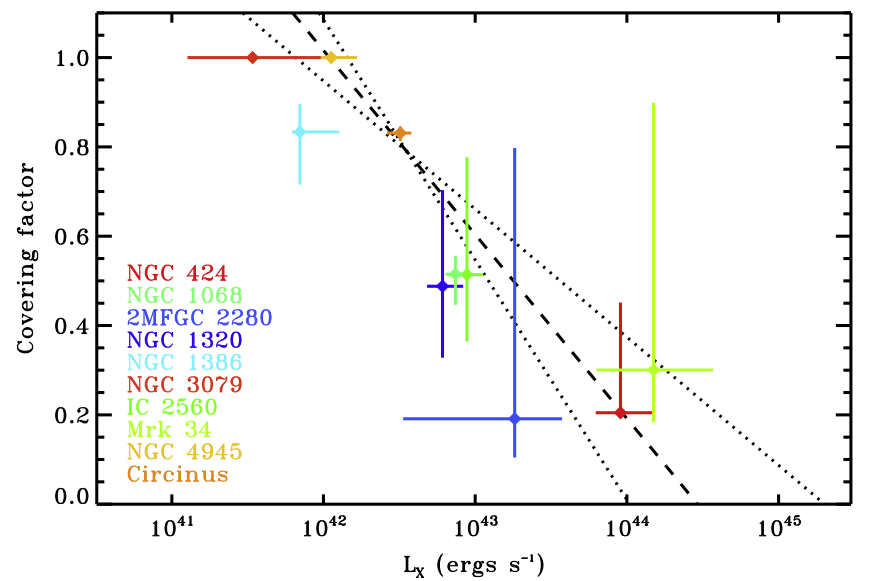

Figure 9. Covering factor as a function of intrinsic $2-10 \mathrm{keV}$ luminosity, $L_{\mathrm{X}}$, derived in our analysis, where the best-fit linear model $f_{\mathrm{c}}=(-0.41 \pm 0.13)$ $\log _{10}\left(L_{\mathrm{X}} / \mathrm{erg} \mathrm{s}^{-1}\right)+18.31 \pm 5.33$ is plotted. NGC 3079 and NGC 4945 are excluded from the linear function fit since they both have no uncertainties on their covering factor. Nonetheless, they both agree well with the derived function.

However, Compton scattering prefers forward (and backward) scattering with small angles, so the difference in light-travel time between transmitted and scattered photons need not be large. The difference in light-travel time is also dependent on the distance of the scatterer from the central source. For the TORUS model, the analysis is limited to a range of covering factors, not allowing investigations of very small covering factors $(\sim 0.1)$ or very high covering factors $(\sim 0.9-0.99)$, and also does not allow one to decouple the transmitted and scattered components. The assumption of a smooth matter distribution is also unlikely to be accurate as it is most likely to be clumpy (Yaqoob 2012). It is clear that further work is required to fully understand the nature of the absorber in NGC 4945.

\section{WHAT DETERMINES THE COVERING FACTOR OF THE OBSCURER IN AGNs?}

The obscurer in local AGNs is widely regarded to be a cold molecular torus, which many recent results imply has a clumpy constituency (e.g., Elitzur \& Shlosman 2006; Markowitz et al. 2014). As discussed, high-energy X-rays are ideally suited to the study of the obscuring material, since Compton scattering effects from the gas in the obscuring medium dominate in this regime $(>10 \mathrm{keV})$. Our fits to $N u S T A R$ data with the TORUS model, which assumes a smooth torus, support the general torus paradigm. In our analysis we determine a wide range of $\theta_{\text {tor }}$ allowed by the model and furthermore identify three sources where the spectral fits indicate small or zero $\theta_{\text {tor. }}$.

Although our sample is small, we investigate what could be physically influencing the opening angle. As the obscured fraction is known to depend on X-ray luminosity, we first investigate how the covering factors derived here depend on $L_{X}$, using the $2-10 \mathrm{keV}$ band and the intrinsic luminosity determined from the model. Figure 9 plots these quantities against each other with their measured uncertainties. A strong anti-correlation is seen, as expected, where one of the most luminous sources, NGC 424 with $\log _{10}\left(L_{X} / \mathrm{erg} \mathrm{s}^{-1}\right)=43.96_{-0.16}^{+0.21}$, has a small, relatively well constrained covering factor $\left(f_{\mathrm{c}}=0.20_{-0.00}^{+0.25}\right)$, while Circinus, with a moderate luminosity of $\log _{10}\left(L_{\mathrm{X}} / \mathrm{erg} \mathrm{s}^{-1}\right)=42.51$ 


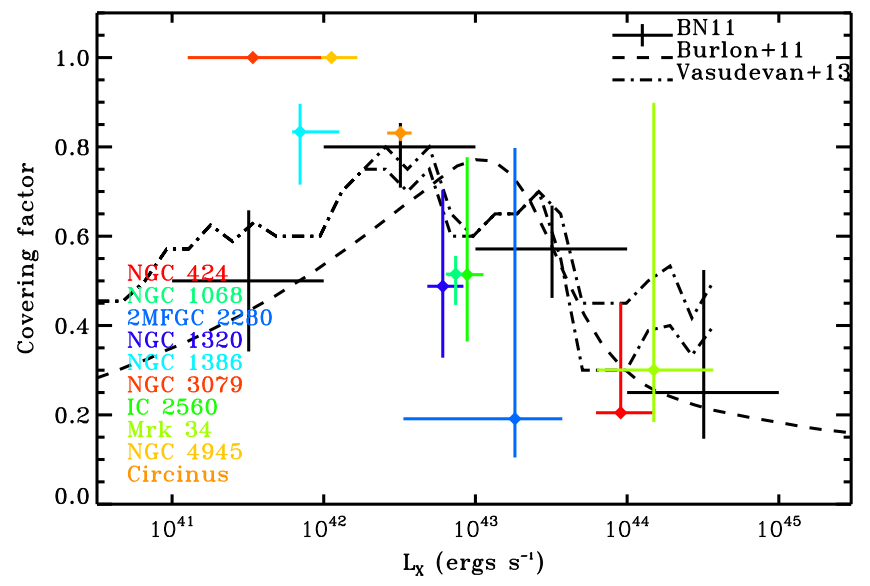

Figure 10. Covering factor of the torus as a function of intrinsic $2-10 \mathrm{keV}$ luminosity, $L_{\mathrm{X}}$, derived in our analysis in comparison to the obscured fraction of local AGN determined from Burlon et al. (2011), Brightman \& Nandra (2011a), and Vasudevan et al. (2013). The covering factors agree well with the obscured fraction for $L_{\mathrm{X}} \gtrsim 10^{42.5} \mathrm{erg} \mathrm{s}^{-1}$. However, at low luminosities, we find no evidence for the decline in the covering factor seen in those studies.

${ }_{-0.09}^{+0.07}$, has a larger covering factor of $f_{\mathrm{c}}=0.83_{-0.02}^{+0.01}$. Such a correlation is expected due to more luminous sources sublimating dust in the inner edge of the torus at larger distances. For the same vertical extension of the torus, a larger radius of the inner part of the torus gives a lower covering factor of the central source due to geometrical effects.

We fit a simple linear model, $y=m x+c$, to the covering factor versus $\log _{10} L_{X}$ data using the IDL function LINFIT, which utilizes $\chi^{2}$ minimisation and takes into account the uncertainties in the covering factor. We find that $f_{\mathrm{c}}=(-0.41 \pm 0.13)$ $\log _{10}\left(L_{\mathrm{X}} / \mathrm{erg} \mathrm{s}^{-1}\right)+18.31 \pm 5.33$, where the uncertainties are $1 \sigma$. We plot this function along with the uncertainties in Figure 9. We do not include the data for NGC 3079 or NGC 4945 in the fit as these have no uncertainties in the covering factors assigned to them. Notably, however, they both agree very well with the fitted model.

We also compare the covering factors to the obscured fraction of local AGNs from three studies. We compare to recent obscured fractions presented by Burlon et al. (2011) and Vasudevan et al. (2013), both hard X-ray-selected samples from Swift/BAT, and that of Brightman \& Nandra (2011b), a mid-infrared, IRAS-selected sample. All three obscured fractions are defined as the fraction of sources with $N_{\mathrm{H}}>10^{22}$ $\mathrm{cm}^{-2}$, calculated in different luminosity bins. We plot these obscured fractions in Figure 10. The uncertainties in the Brightman \& Nandra (2011b) data points are binomial. The Burlon et al. (2011) curve is calculated by dividing the X-ray luminosity function (XLF) of obscured AGNs by the total XLF, done in the $15-55 \mathrm{keV}$ band. The Vasudevan et al. (2013) line is a running average using 30 sources per bin. The two different lines for this sample are derived from where there are uncertainties on the $N_{\mathrm{H}}$ measurement, and the upper $N_{\mathrm{H}}$ bound is used for the upper line and the lower $N_{\mathrm{H}}$ bound is used for the lower line.

The obscured fractions determined in these three studies agree very well with each other despite the differing selections and determinations, declining from a peak at $L_{\mathrm{X}}=10^{42-43} \mathrm{erg} \mathrm{s}^{-1}$ toward higher luminosities. All three studies also find evidence for a decline in the obscured fraction toward lower luminosities.

Also in Figure 10, we overplot the covering factors derived from our sample. We find good agreement between our derived covering factors and the obscured fraction for $L_{\mathrm{X}} \gtrsim 10^{42.5} \mathrm{erg} \mathrm{s}^{-1}$. However, while previous studies have found evidence for a decrease in the covering factor at low luminosities, we find that our sources in the $L_{\mathrm{X}}=10^{41-42} \mathrm{erg} \mathrm{s}^{-1}$ range are heavily buried in material with high covering factors. A larger, more complete sample is required to show if this disagreement is statistically significant or due to the low number statistics of our sample.

We note that the covering factors derived here are those of the CT gas surrounding the AGNs, since the TORUs model assumes a constant density torus with a constant $N_{\mathrm{H}}$ as a function of inclination angle. Therefore, any additional covering by Compton-thin gas would not be recognized, in which case the covering factors here could underestimate the total covering factor. Due to the agreement between the CT covering factor and the obscured fraction (for $L_{\mathrm{X}}>10^{42.5} \mathrm{erg} \mathrm{s}^{-1}$ ), this does not appear to be the case in our sample. This implies uniform torus covering factors for Compton-thin and CT AGNs given the same $L_{\mathrm{X}}$, above $10^{42.5} \mathrm{erg} \mathrm{s}^{-1}$. Best estimates of the local CT fraction put it at $20 \%$ (Burlon et al. 2011; Brightman \& Nandra 2011b), which is lower than the covering factors determined here, which could suggest that a larger population of CT AGNs exists in the local universe.

Lastly, we briefly investigate what other AGNs parameters may be involved in determining the covering factor of the obscurer. We have already shown that the X-ray luminosity, which traces the bolometric power of the AGNs and is thus dependent on the mass accretion rate, plays an important role. We next explore if the mass of the black hole, $M_{\mathrm{BH}}$, or the fraction of the Eddington luminosity, $\lambda_{\text {Edd }}$, physically influences the covering factor. These quantities are notoriously difficult to determine in obscured AGNs, as virial mass estimates from optical broad lines are not accessible. In these cases, the velocity dispersion of the stars in the bulge is often used to estimate $M_{\mathrm{BH}}$ from the $M_{\mathrm{BH}}-\sigma_{*}$ relation, although there is evidence for large scatter in this relationship, especially at low mass (Greene et al. 2010). For some sources, water megamasers can provide robust black hole mass measurements. We assemble these data from the literature and list them in Table 6. Some $\lambda_{\text {Edd }}$ estimates are also available. The covering factors of the torus relative to these quantities are plotted in Figure 11.

This preliminary investigation seems to show that the highest black hole mass systems in our sample have the smallest covering factor, while the smallest black holes have the highest covering factors; however, the relationship is not statistically significant and a far larger sample is required to confirm this trend and to break the degeneracy with $L_{\mathrm{X}}$. As for the covering factor as a function of $\lambda_{\text {Edd }}$, our data suggest that high covering factors are exhibited in both low $\left(10^{-3}\right)$ and high $(\sim 0.3)$ $\lambda_{\text {Edd }}$ systems, in line with the conclusions of Draper \& Ballantyne (2010), who find that CT AGNs are made up of a composite population of both high $(>0.9)$ and low $(<0.01)$ Eddington ratio systems.

\section{CONCLUSIONS}

In this paper we have used the X-ray torus models of Brightman \& Nandra (2011a) and data from NuSTAR and 
Table 6

Accretion Parameters of Our Sample

\begin{tabular}{|c|c|c|c|c|c|c|}
\hline $\begin{array}{l}\text { Source Name } \\
\text { (1) }\end{array}$ & $\begin{array}{c}\text { Class } \\
(2)\end{array}$ & $\begin{array}{l}f_{\mathrm{c}} \\
(3)\end{array}$ & $\begin{array}{c}\log _{10} L_{X} \\
(4)\end{array}$ & $\begin{array}{c}\log _{10} M_{\mathrm{BH}} \\
(5)\end{array}$ & $\begin{array}{c}\log _{10} \lambda_{\text {Edd }} \\
(6)\end{array}$ & $\begin{array}{c}\text { References } \\
(7)\end{array}$ \\
\hline NGC 424 & Sylh & $0.20_{-0.01}^{+0.25}$ & $43.96_{-0.16}^{+0.21}$ & 7.78 & -1.30 & 1,6 \\
\hline NGC 1068 & Sylh & $0.52_{-0.07}^{+0.04}$ & $42.87{ }_{-0.06}^{+0.04}$ & 7.59 & -1.42 & 2,2 \\
\hline 2MFGC 2280 & Sy2 & $0.19{ }_{-0.09}^{+0.61}$ & $43.26_{-0.74}^{+0.31}$ & 0.00 & -99.00 & 0,0 \\
\hline NGC 1320 & Sy2 & $0.50_{-0.17}^{+0.21}$ & $42.79_{-0.09}^{+0.12}$ & 7.29 & -1.54 & 2,2 \\
\hline NGC 1386 & Sy1i & $0.83_{-0.12}^{+0.06}$ & $41.84_{-0.05}^{+0.26}$ & 7.42 & -2.92 & 2,2 \\
\hline Mrk 34 & Sy2 & $0.30_{-0.12}^{+0.60}$ & $44.18_{-0.38}^{+0.39}$ & 7.90 & -99.00 & 5,0 \\
\hline NGC 4945 & FSRS & $1.00_{-0.00}^{+0.00}$ & $41.92_{-0.08}^{+0.07}$ & 6.15 & -99.00 & 4,0 \\
\hline Circinus & Sy1h & $0.83_{-0.02}^{+0.01}$ & $42.51{ }_{-0.09}^{+0.07}$ & 6.18 & -0.70 & 8,9 \\
\hline
\end{tabular}

Note. Column (1) lists the source name, column (2) gives the AGN activity class based on the optical spectrum, where Sylh indicates a Seyfert 2 with a hidden broadline region revealed in polarized light, Syli indicates a Seyfert 2 with a broad-line region visible in the infrared, Sy2 indicates a Seyfert 2 with no evidence for a hidden broad-line region, and FSRS means a flat-spectrum radio source. Column (3) gives the covering factor of the obscuring material derived in this work, column (4) gives $\log _{10}\left(L_{X} / \mathrm{erg} \mathrm{s}^{-1}\right)$ also derived here, column (5) lists the black hole masses from the literature in logarithm of solar masses, column (6) gives the Eddington ratio published in the literature, and column (7) lists the references for these data: 1. Bian \& Gu (2007) (black hole mass from stellar velocity dispersion), 2. Marinucci et al. (2012) (black hole mass from stellar velocity dispersion), 3. Ishihara et al. (2001) (black hole mass from water megamaser emission), 4. Greenhill et al. (1997) (black hole mass from water megamaser emission), 5. Su et al. (2008) (black hole mass from [O III] line width), 6. Baloković et al. (2014), 7. Kondratko et al. (2005), 8. Greenhill et al. (2003) (black hole mass from water megamaser emission), 9. Arévalo et al. (2014).

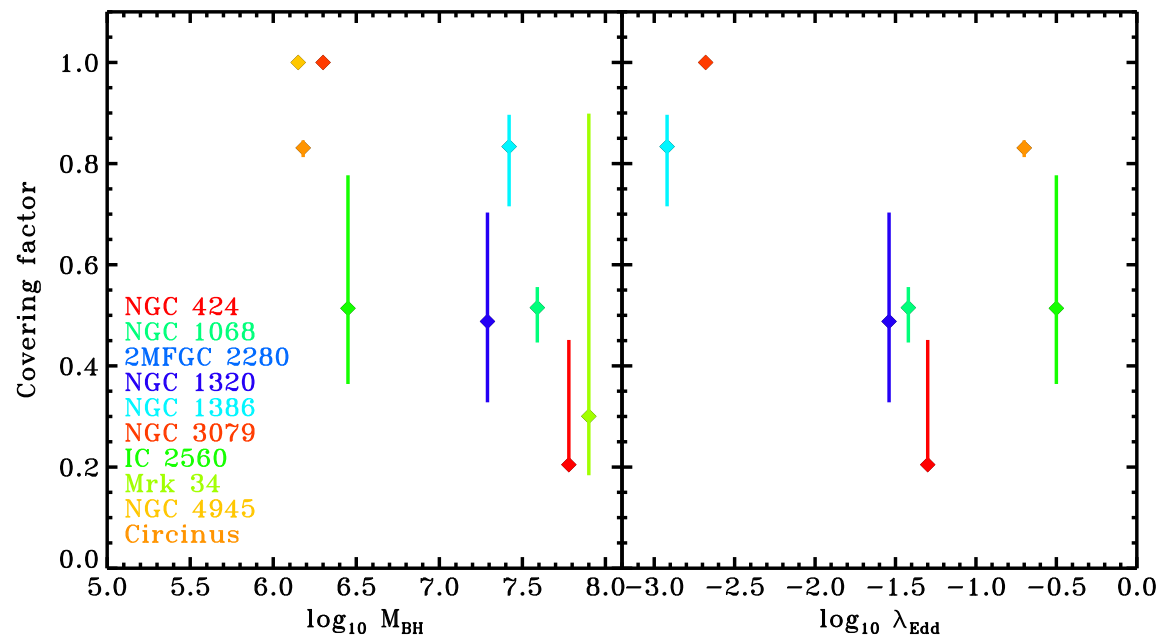

Figure 11. Our derived covering factor of the obscuring material compared to the black hole masses (in solar masses) and the Eddington ratios from the literature.

$X M M-N e w t o n$ to determine the covering factor of the CT gas in 10 local CT AGNs, NGC 424, NGC 1068, 2MFGC 2280, NGC 1320, NGC 1386, IC 2560, Mrk 34, NGC 3079, NGC 4945, and Circinus. We have also assessed the differences between the TORUS model, PEXRAV, and MYTORUS. We find:

1. The slab reflection model, PEXRAv, does not easily reproduce the Compton hump shape produced by a torus geometry, underproducing it for large covering factors, and overproducing it for small ones, resulting in a systematic offset in the parameters obtained. We therefore discourage use of this model in the fitting of highenergy X-ray emission of CT AGNs. Our results compare well with MYTORUS for $N_{\mathrm{H}}<10^{25} \mathrm{~cm}^{-2}$, where that model is valid; however, we support the use of the covering factor as a free parameter in torus models.

2. Measurements of $\theta_{\text {tor }}$ are in the range of $26^{\circ}-80^{\circ}$, limited by the allowed range of the model, with uncertainties on these measurements ranging from $5^{\circ}$ to $40^{\circ}$. These correspond to covering factors in the range of 0.2-0.9, with uncertainties ranging from 0.05 to 0.6 .

3. The covering factor is a strongly decreasing function of intrinsic 2-10 keV luminosity; when fitted with a linear function, we find $f_{\mathrm{c}}=(-0.41 \pm 0.13) \log _{10}$ $\left(L_{\mathrm{X}} / \mathrm{erg} \mathrm{s}^{-1}\right)+18.31 \pm 5.33$.

4. The individual covering factors derived here agree well with the average covering factor of local AGNs as measured by the obscured fraction as a function of $L_{\mathrm{X}}$ above $10^{42.5} \mathrm{erg} \mathrm{s}^{-1}$. However, while previous studies have found evidence for a decrease in the covering factor at low luminosities, we find that our sources in the $L_{\mathrm{X}}=10^{41-42} \mathrm{erg} \mathrm{s}^{-1}$ range are heavily buried in material with high covering factors. A larger, more complete sample is required to show if this disagreement is statistically significant or due to the low number statistics of our sample.

5. We find a conflicting result on NGC 4945, where our spectral analysis implies a large, almost $100 \%$, covering 
factor, whereas previous results have concluded that this source has a very low covering factor due to flux variability above $10 \mathrm{keV}$. We conclude that model limitations in both cases are the likely cause of the disagreement.

This work was supported under NASA Contract No. NNG08FD60C and made use of data from the NuSTAR mission, a project led by the California Institute of Technology, managed by the Jet Propulsion Laboratory, and funded by the National Aeronautics and Space Administration. We thank the NuSTAR Operations, Software, and Calibration teams for support with the execution and analysis of these observations. This research has made use of the NuSTAR Data Analysis Software (NuSTARDAS) jointly developed by the ASI Science Data Center (ASDC, Italy) and the California Institute of Technology (USA). The work presented here was also based on observations obtained with XMM-Newton, an ESA science mission with instruments and contributions directly funded by ESA Member States and NASA. This research has also made use of data and software provided by the High Energy Astrophysics Science Archive Research Center (HEASARC), which is a service of the Astrophysics Science Division at NASA/GSFC and the High Energy Astrophysics Division of the Smithsonian Astrophysical Observatory. Furthermore, this research has made use of the NASA/IPAC Extragalactic Database (NED), which is operated by the Jet Propulsion Laboratory, California Institute of Technology, under contract with the National Aeronautics and Space Administration. M. Baloković acknowledges support from NASA Headquarters under the NASA Earth and Space Science Fellowship Program, grant NNX14AQ07H. D.R.B. acknowledges support from NSF award AST 1008067; P.G. acknowledges support from STFC (grant reference ST/J003697/1), M.K. acknowledges support from the Swiss National Science Foundation (SNSF) through the Ambizione fellowship grant PZ00P2 154799/1. We also acknowledge support from CONICYT-Chile grants BasalCATA PFB-06/2007 (FEB), FONDECYT 1141218 (FEB), "EMBIGGEN" Anillo ACT1101 (FEB), Project IC120009 "Millennium Institute of Astrophysics (MAS)" of the Iniciativa Científica Milenio del Ministerio de Economía, Fomento y Turismo (FEB).

Facilities: NuSTAR, XMM (EPIC)

\section{REFERENCES}

Akylas, A., \& Georgantopoulos, I. 2009, A\&A, 500, 999

Akylas, A., Georgantopoulos, I., Georgakakis, A., Kitsionas, S., \& Hatziminaoglou, E. 2006, A\&A, 459, 693

Anders, E., \& Grevesse, N. 1989, GeCoA, 53, 197

Arévalo, P., Bauer, F. E., Puccetti, S., et al. 2014, ApJ, 791, 81

Assef, R. J., Stern, D., Kochanek, C. S., et al. 2013, ApJ, 772, 26

Awaki, H., Terashima, Y., Higaki, Y., \& Fukazawa, Y. 2009, PASJ, 61, 317

Ballantyne, D. R., Bollenbacher, J. M., Brenneman, L. W., et al. 2014, ApJ, 794, 62

Baloković, M., Comastri, A., Harrison, F. A., et al. 2014, ApJ, 794, 111

Baloković, M., Matt, G., Harrison, F. A., et al. 2015, ApJ, 800, 62
Bambynek, W., Crasemann, B., Fink, R. W., et al. 1972, RvMP, 44, 716 Bauer, F. E., Arevalo, P., Walton, D. J., et al. 2014, arXiv:1411.0670 Beckmann, V., Soldi, S., Ricci, C., et al. 2009, A\&A, 505, 417 Bian, W., \& Gu, Q. 2007, ApJ, 657, 159

Brenneman, L. W., Madejski, G., Fuerst, F., et al. 2014, ApJ, 788, 61 Brightman, M., \& Nandra, K. 2011, MNRAS, 413, 1206

Brightman, M., \& Nandra, K. 2011, MNRAS, 414, 3084

Brightman, M., Nandra, K., Salvato, M., et al. 2014, MNRAS, 443, 1999

Brightman, M., \& Ueda, Y. 2012, MNRAS, 423, 702

Buchner, J., Georgakakis, A., Nandra, K., et al. 2014, A\&A, 564, A125

Burlon, D., Ajello, M., Greiner, J., et al. 2011, ApJ, 728, 58

Comastri, A., Ranalli, P., Iwasawa, K., et al. 2011, A\&A, 526, L9

Done, C., Madejski, G. M., Życki, P. T., \& Greenhill, L. J. 2003, ApJ, 588, 763

Draper, A. R., \& Ballantyne, D. R. 2010, ApJL, 715, L99

Eguchi, S., Ueda, Y., Awaki, H., et al. 2011, ApJ, 729, 31

Elitzur, M., \& Shlosman, I. 2006, ApJL, 648, L101

Gandhi, P., Horst, H., Smette, A., et al. 2009, A\&A, 502, 457

Gandhi, P., Lansbury, G. B., Alexander, D. M., et al. 2014, ApJ, 792, 117

Ghisellini, G., Haardt, F., \& Matt, G. 1994, MNRAS, 267, 743

Goulding, A. D., Alexander, D. M., Bauer, F. E., et al. 2012, ApJ, 755, 5

Greene, J. E., Peng, C. Y., Kim, M., et al. 2010, ApJ, 721, 26

Greenhill, L. J., Kondratko, P. T., Lovell, J. E. J., et al. 2003, ApJL, 582, L11

Greenhill, L. J., Moran, J. M., \& Herrnstein, J. R. 1997, ApJL, 481, L23

Gu, M. 2013, ApJ, 773, 176

Guainazzi, M., \& Bianchi, S. 2007, MNRAS, 374, 1290

Harrison, F. A., Craig, W. W., Christensen, F. E., Hailey, C. J., \& Zhang, W. W. 2013, ApJ, 770, 103

Hasinger, G. 2008, A\&A, 490, 905

Ichikawa, K., Packham, C., Ramos Almeida, C., et al. 2015, arXiv:1501.06584 Ikeda, S., Awaki, H., \& Terashima, Y. 2009, ApJ, 692, 608

Ishihara, Y., Nakai, N., Iyomoto, N., et al. 2001, PASJ, 53, 215

Kawamuro, T., Ueda, Y., Tazaki, F., \& Terashima, Y. 2013, ApJ, 770, 157

Kondratko, P. T., Greenhill, L. J., \& Moran, J. M. 2005, ApJ, 618, 618

La Franca, F., Fiore, F., Comastri, A., et al. 2005, ApJ, 635, 864

Lawrence, A. 1991, MNRAS, 252, 586

Lawrence, A., \& Elvis, M. 2010, ApJ, 714, 561

Leahy, D. A., \& Creighton, J. 1993, MNRAS, 263, 314

Liu, Y., \& Li, X. 2014, ApJ, 787, 52

Lusso, E., Hennawi, J. F., Comastri, A., et al. 2013, ApJ, 777, 86

Madejski, G., Życki, P., Done, C., et al. 2000, ApJL, 535, L87

Magdziarz, P., \& Zdziarski, A. A. 1995, MNRAS, 273, 837

Marinucci, A., Bianchi, S., Nicastro, F., Matt, G., \& Goulding, A. D. 2012, ApJ, 748, 130

Marinucci, A., Matt, G., Kara, E., et al. 2014, MNRAS, 440, 2347

Markowitz, A. G., Krumpe, M., \& Nikutta, R. 2014, MNRAS, 439, 1403

Matt, G., Perola, G. C., \& Piro, L. 1991, A\&A, 247, 25

Mayo, J. H., \& Lawrence, A. 2013, MNRAS, 434, 1593

Molendi, S., Bianchi, S., \& Matt, G. 2003, MNRAS, 343, L1

Murphy, K. D., \& Yaqoob, T. 2009, MNRAS, 397, 1549

Nandra, K., \& George, I. M. 1994, MNRAS, 267, 974

Puccetti, S., Comastri, A., Fiore, F., et al. 2014, ApJ, 793, 26

Risaliti, G., Maiolino, R., \& Salvati, M. 1999, ApJ, 522, 157

Sambruna, R. M., Netzer, H., Kaspi, S., et al. 2001, ApJL, 546, L13

Simpson, C. 2005, MNRAS, 360, 565

Su, J.-B., Zhang, J.-S., \& Fan, J.-H. 2008, ChJAA, 8, 547

Tazaki, F., Ueda, Y., Terashima, Y., \& Mushotzky, R. F. 2011, ApJ, 738, 70 Tilak, A., Greenhill, L. J., Done, C., \& Madejski, G. 2008, ApJ, 678, 701

Treister, E., Krolik, J. H., \& Dullemond, C. 2008, ApJ, 679, 140

Tueller, J., Mushotzky, R. F., Barthelmy, S., et al. 2008, ApJ, 681, 113

Ueda, Y., Akiyama, M., Ohta, K., \& Miyaji, T. 2003, ApJ, 598, 886

Vasudevan, R. V., Brandt, W. N., Mushotzky, R. F., et al. 2013, ApJ, 763, 111

Verner, D. A., Ferland, G. J., Korista, K. T., \& Yakovlev, D. G. 1996, ApJ, 465,487

Walton, D. J., Fuerst, F., Harrison, F., et al. 2013, ApJ, 779, 148

Walton, D. J., Harrison, F. A., Grefenstette, B. W., et al. 2014, ApJ, 793, 21

Yaqoob, T. 1997, ApJ, 479, 184

Yaqoob, T. 2012, MNRAS, 423, 3360 